Article

\title{
Chirvinskyite, $(\mathrm{Na}, \mathrm{Ca})_{13}(\mathrm{Fe}, \mathrm{Mn}, \mathrm{D})_{2}(\mathrm{Ti}, \mathrm{Nb})_{2}(\mathrm{Zr}, \mathrm{Ti})_{3}-$ $\left(\mathrm{Si}_{2} \mathrm{O}_{7}\right)_{4}(\mathrm{OH}, \mathrm{O}, \mathrm{F})_{12}$, a New Mineral with a Modular Wallpaper Structure, from the Khibiny Alkaline Massif (Kola Peninsula, Russia)
}

\author{
Victor N. Yakovenchuk ${ }^{1,2}$, Yakov A. Pakhomovsky ${ }^{1,2}$, Taras L. Panikorovskii ${ }^{3}{ }^{(0}$, \\ Andrey A. Zolotarev ${ }^{4}$, Julia A. Mikhailova ${ }^{1,2}$, Vladimir N. Bocharov ${ }^{5}{ }^{\circ}$, \\ Sergey V. Krivovichev $1,4, *$ (i) and Gregory Yu. Ivanyuk ${ }^{1,2}$ \\ 1 Nanomaterials Research Centre of Kola Science Centre, Russian Academy of Sciences, 14 Fersman Street, \\ Apatity 184209, Russia; yakovenchuk@geoksc.apatity.ru (V.N.Y.); pakhom@geoksc.apatity.ru (Y.A.P.); \\ ylya_korchak@mail.ru (J.A.M.); ivanyuk@admksc.apatity.ru (G.Y.I.) \\ 2 Geological Institute of Kola Science Centre, Russian Academy of Sciences, 14 Fersman Street, \\ Apatity 184209, Russia \\ 3 Laboratory of geo-inspired technologies and environmental safety of Arctic region of Kola Science Centre, \\ Russian Academy of Sciences, 14 Fersman Street, Apatity 184209, Russia; taras.panikorovsky@spbu.ru \\ 4 Department of Crystallography, Institute of Earth Sciences, Saint-Petersburg State University, University \\ Emb. 7/9, St. Petersburg 199034, Russia; aazolotarev@gmail.com \\ 5 Geo Environmental Centre “Geomodel”, Saint-Petersburg State University, Ul'yanovskaya Str. 1, \\ St. Petersburg 198504, Russia; bocharov@molsp.phys.spbu.ru \\ * Correspondence: s.krivovichev@spbu.ru; Tel.: +7-81555-79321
}

Received: 17 March 2019; Accepted: 4 April 2019; Published: 6 April 2019

Abstract: Chirvinskyite, $(\mathrm{Na}, \mathrm{Ca})_{13}(\mathrm{Fe}, \mathrm{Mn}, \square)_{2}(\mathrm{Ti}, \mathrm{Nb})_{2}\left(\mathrm{Zr}, \mathrm{Ti}_{3}\left(\mathrm{Si}_{2} \mathrm{O}_{7}\right)_{4}(\mathrm{OH}, \mathrm{O}, \mathrm{F})_{12}\right.$, is a new wöhleriterelated zirconotitano-sorosilicate. It is triclinic, $P \overline{1}, a=7.0477(5), b=9.8725(5), c=12.2204(9) \AA$, $\alpha=77.995(5), \beta=82.057(6), \gamma=89.988(5)^{\circ}, V=823.35(9) \AA^{3}, Z=1$. The mineral was found in albitized alkaline pegmatites in a foyaite of the Mt. Takhtarvumchorr (Khibiny alkaline massif, Kola Peninsula, Russia, N $67^{\circ} 40^{\prime}$, E $33^{\circ} 33^{\prime}$ ). Chirvinskyite forms sheaf-like and radiated aggregates (up to $6 \mathrm{~mm}$ in diameter) of split fibrous crystals hosted by saccharoidal fluorapatite and albite. The mineral is pale cream in color, with a silky luster and a white streak. The cleavage is not recognized. Mohs hardness is 5. Chirvinskyite is biaxial (-), $\alpha$ 1.670(2), $\beta 1.690(2), \gamma 1.705(2)(589 \mathrm{~nm})$, $2 V_{\text {calc }}=80.9^{\circ}$. The calculated and measured densities are 3.41 and $3.07(2) \mathrm{g} \cdot \mathrm{cm}^{-3}$, respectively. The empirical formula based on $\mathrm{Si}=8$ apfu is $\left(\mathrm{Na}_{9.81} \mathrm{Ca}_{3.28} \mathrm{~K}_{0.01}\right)_{\sum 13.10}\left(\mathrm{Fe}_{0.72} \mathrm{Mn}_{0.69} \square_{0.54} \mathrm{Mg}_{0.05}\right)_{\sum 2.00}$ $\left(\mathrm{Ti}_{1.81} \mathrm{Nb}_{0.19}\right)_{\sum 2.00}\left(\mathrm{Zr}_{2.27} \mathrm{Ti}_{0.63}\right)_{\sum 2.90}\left(\mathrm{Si}_{2} \mathrm{O}_{7}\right)_{4}\left\{(\mathrm{OH})_{5.94} \mathrm{O}_{3.09} \mathrm{~F}_{2.97}\right\}_{\sum 12.00}$. Chirvinskyite belongs to a new structure type of minerals and inorganic compounds and is related to the wöhlerite-group minerals. Its modular "wallpaper" structure consists of disilicate groups $\mathrm{Si}_{2} \mathrm{O}_{7}$ and three types of "octahedral walls". The mineral is named in honor of Petr Nikolaevich Chirvinsky (1880-1955), Russian geologist and petrographer, head of the Petrography Department of the Perm' State University (1943-1953), for his contributions to mineralogy and petrology, including studies of the Khibiny alkaline massif.

Keywords: chirvinskyite; new mineral; titanozircono-sorosilicate; modular crystal structure; albitite; Khibiny massif

\section{Introduction}

Zircono- and titanosilicates of the wöhlerite family are characteristic minerals of the alkaline complexes, including the Khibiny massif in Kola Peninsula, Russia. These minerals have complex 
modular crystal structures formed by $\mathrm{Si}_{2} \mathrm{O}_{7}$ groups and complex zigzag octahedral walls similar to those observed in borates with wallpaper structures [1,2]. The chemical composition and configuration of these walls vary widely [3,4], which results in about 10 approved wöhlerite-related minerals, with five of them (wöhlerite, låvenite, normandite, cuspidine, and chirvinskyite) occuring in the Khibiny massif [5].

The thinnest split fibers of an unknown låvenite-like mineral were found by late Yuri Men'shikov (1934-2013) during an investigation of the apatite/molybdenite/parakeldyshite deposit at Mt. Takhtarvumchorr [5]. Men'shikov obtained chemical and powder XRD data for this mineral and proposed to name it in honor of Prof. Petr Nikolaevich Chirvinsky (1880-1955), Russian geologist and petrographer, head of the Petrography Department of the Perm State University (1943-1953), for his contributions to mineralogy and petrology, including studies of the Khibiny alkaline massif in 1931-1941 [6,7]. However, the quality of crystals available at the time did not allow solution of the crystal structure of the mineral, which was necessary for its approval as a new mineral species. In 2015, we collected much larger radiated aggregates of this mineral in albitized alkaline pegmatites of the same locality, which allowed us to solve its crystal structure and finish the chirvinskyite investigation.

Both the mineral and mineral name have been approved by the Commission on New Minerals, Nomenclature, and Classification of the International Mineralogical Association (IMA 2016-051). Type material is deposited in the collections of the Mineralogical Museum of St. Petersburg State University, Russia, under catalogue number 19657 and in the Geological and Mineralogical Museum of the Geological Institute of the Kola Science Centre, Apatity, Russia, under catalogue number GIM 7609.

\section{Materials and Methods}

The mineral was found and investigated in hand-sized specimens collected in 2000-2015 for most investigations. Fragments of fibrous chirvinskyite aggregates (up to $4 \times 2 \times 2 \mathrm{~mm}^{3}$ ) were used.

Optical properties of chirvinskyite were determined by using an Amplival optical microscope (Carl Zeiss Microscopy, Jena, Germany) and standard immersion liquids. The mineral density was determined by the float-sink method in Clerici solution (equal parts of $\mathrm{TlHCO}_{2}$ and $\mathrm{TlC}_{3} \mathrm{H}_{3} \mathrm{O}_{4}$ plus water), with the liquid density estimated by its refractive index.

The identification of associated minerals as well as the study of chirvinskyite morphology were performed using a LEO-1450 scanning electron microscope (Carl Zeiss Microscopy, Oberkochen, Germany) with a Quantax 200 energy-dispersive X-ray spectrometer (Bruker, Ettlingen, Germany).

The chemical composition of chirvinskyite was determined by wavelength-dispersive spectrometry on a Cameca MS-46 electron microprobe (Geological Institute, Kola Science Center, Russian Academy of Sciences, Apatity, Russia) operating at 20 kV, 20-30 nA. The $\mathrm{H}_{2} \mathrm{O}$ content was calculated from the structural data and confirmed by the Penfield method [8] for the hand-cleaned material. Other elements with atomic numbers exceeding 5 were not detected. The analyses were performed with the beam size of $5 \mu \mathrm{m}$ and the counting time of 10-20/10 s on peaks/background for every chemical element. The standards used included fluorite $(\mathrm{F})$, lorenzenite $(\mathrm{Na})$, pyrope $(\mathrm{Mg})$, diopside (Si, Ca), wadeite $(\mathrm{K})$, rutile (Ti), synthetic $\mathrm{MnCO}_{3}(\mathrm{Mn})$, hematite $(\mathrm{Fe})$, synthetic $\mathrm{ZrSiO}_{4}(\mathrm{Zr})$, and $\mathrm{LiNbO}_{3}(\mathrm{Nb})$. The coefficients in crystal chemical formulas were calculated by using the MINAL program [9].

The Raman spectrum of chirvinskyite was measured using a Jobin-Yvon LabRam HR 800 spectrometer (Horiba, Kyoto, Japan) with a 514 nm laser.

The powder X-ray diffraction pattern of chirvinskyite was obtained using a Rigaku R-AXIS RAPID II diffractometer equipped with a cylindrical image plate detector, with the Debye-Scherrer geometry ( $d=127.4 \mathrm{~mm}, \mathrm{CoK} \alpha$ radiation). The data were integrated using the software package OSC2XRD [10]. Unit-cell dimensions were determined from the corrected X-ray powder-diffraction by Rietveld refinement using the program Topas [11].

The single-crystal X-ray diffraction study was performed using the Bruker Kappa APEX DUO diffractometer operated at $45 \mathrm{kV}$ and $0.6 \mathrm{~mA}$ and equipped with a CCD area detector by means of a monochromatic MoK $\alpha$ X-radiation, frame widths of $1^{\circ}$ in $\omega$, and $200 \mathrm{~s}$ counting time for each frame. 
The intensity data were reduced and corrected for Lorentz, polarization, and background effects using the Bruker software APEX2 [12]. A semi-empirical absorption-correction based upon the intensities of equivalent reflections was applied $[13,14]$. The structure was solved and refined with the ShelX program package $[14,15]$ within the Olex2 shell [16] to $R_{1}=0.0736$ for 3826 independent reflections with $F^{2}>4 \sigma\left(F^{2}\right)$. Occupancies of the cation sites were calculated from the experimental site-scattering factors considering empirical chemical composition. Images of the chirvinskyite crystal structure were drawn with the "Atoms 6.5" program [17]. Further details about the crystal structure of the mineral are provided in the Crystallographic Information File (CIF) available as a Supplementary Information.

\section{Results}

\subsection{Occurrence}

The Khibiny alkaline massif (Kola Peninsula, Russia) is the world largest alkaline complex that was protruded through the Archaean granite-gneisses, Proterozoic amphibole schists, and Devonian traps about $370 \mathrm{Ma}$ [18]. The stock-like foyaite protrusion (about $30 \mathrm{~km}$ in diameter) was sharply expanded near the day surface (to about $50 \mathrm{~km}$ in diameter) with the formation of ring faults filled by residual foidolite melts (Figure 1a).
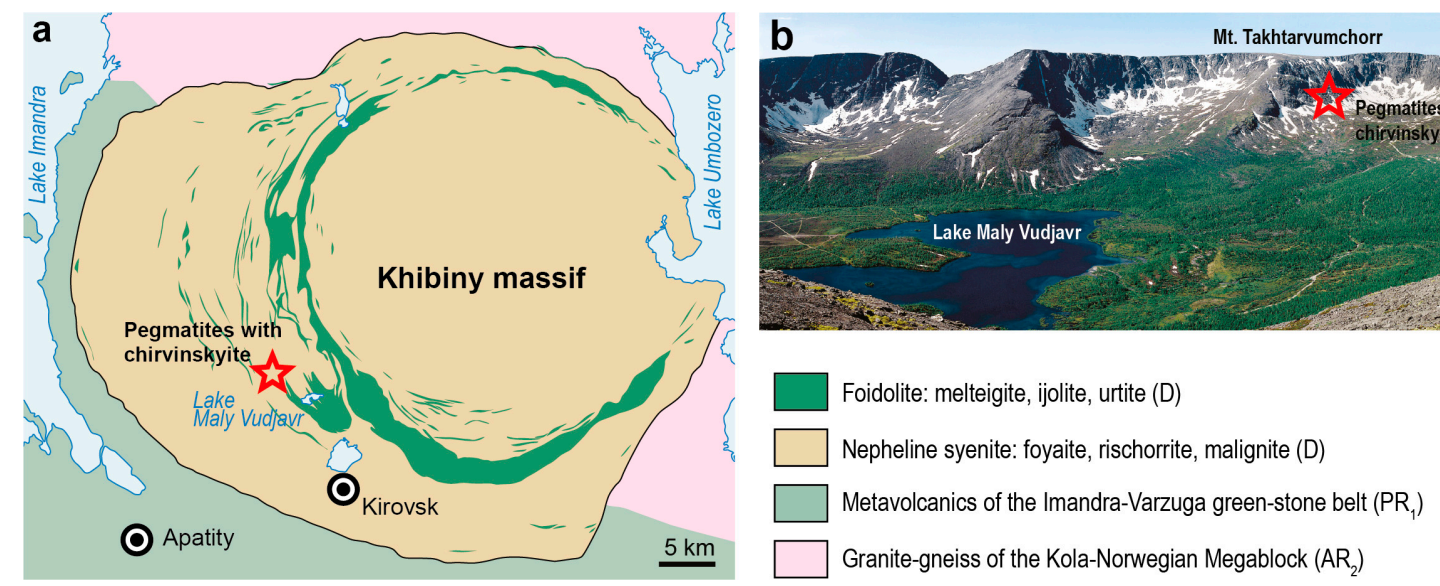

Figure 1. Geology of the Khibiny massif (a) and type-locality of chirvinskyite marked by the star (b).

Numerous xenoliths of olivine basalt and tuff interlayered with quartzite and sandstone are widespread within the foyaite massif. They are comparatively fresh far from the foidolite rings and strongly fenitized near the rings [19]. Fenitization of the basalt and tuff produced Ca-Ti-rich hydrothermal solutions that made surrounding foyaites rich in $\mathrm{Ca}$ and $\mathrm{Ti}$ with the formation of corresponding mineral assemblages (fluorapatite, titanite, rinkite, rosenbushite, etc.) including minerals of the wöhlerite group: cuspidine, låvenite, normandite, wöhlerite [5], and chirvinskyite.

The type locality of chirvinskyite (Figure 1b) is situated at the Mt. Takhtarvumchorr near the large xenolith of deeply fenitized basaltic tuff consisting mainly of orthoclase, cordierite, phlogopite, and pyrrhotite. Surrounding host foyaite is intensively albitized and contains fluorapatite, molybdenite, graphite, and zircon-parakeldyshite mineralization of minor economic significance [5,6]. Numerous veins of albitized/natrolitized nepheline-microcline-aegirine-augite pegmatites (up to $6 \mathrm{~m}$ wide and $200 \mathrm{~m}$ long) are abundant in this region (localities nos. 53 and 54 in [5]), and chirvinskyite occurs in one of the veins $\left(\mathrm{N} 67^{\circ} 40^{\prime}, \mathrm{E} 33^{\circ} 33^{\prime}\right)$.

Chirvinskyite was found in eluvial blocks of a thick (above $1 \mathrm{~m}$ ) albitized and natrolitized pegmatite with indistinct contact with the host foyaite (due to intensive metasomatic alteration of both pegmatite and the host rock). The pegmatite blocks consist mainly of white fine-grained albite with corroded microcline laths (up to $3 \mathrm{~cm}$ long), fine-grained natrolite replacing nepheline, prismatic crystals of aegirine-augite and arfvedsonite (up to $4 \mathrm{~cm}$ long), flattened prismatic crystals of 
astrophyllite (up to $5 \mathrm{~cm}$ long), radiating and stream-like aggregates of black needle-like crystals of aegirine (up to $2 \mathrm{~cm}$ long), and relics of altered light brown eudialyte (up to $1 \mathrm{~cm}$ in diameter). Aegirine aggregates are often impregnated with thin graphite lamella. In the axial zones of pegmatite, there are lens-shaped clusters (up to $1 \times 0.50 \mathrm{~m}$ ) of light-green saccharoidal fluorapatite with inclusions of flattened prismatic crystals of aenigmatite (up to $3 \mathrm{~cm}$ long), lamellar crystals of molybdenite, ilmenite, and pyrrhotite (up to $7 \mathrm{~mm}$ in diameter), which is partially replaced by pyrite, marcasite, and goethite, rounded grains of galena (up to $5 \mathrm{~mm}$ ) and sphalerite (up to $1 \mathrm{~cm}$ ), and reddish-orange prismatic crystals of låvenite (up to $3 \mathrm{~mm}$ long). In places, there are lemon-yellow radiating aggregates (up to $4 \mathrm{~cm}$ in diameter) and prismatic crystals (up to $1.5 \mathrm{~cm}$ long) of titanite, partially replaced by lorenzenite, as well as rich impregnations of eudialyte and zircon grains (up to $6 \mathrm{~mm}$ ) surrounded by 1-3 mm wide snow-white fringes of parakeldyshite, sodalite, and cancrinite [5].

Chirvinskyite forms sheaf-like and radiating aggregates up to $6 \mathrm{~mm}$ in diameter (Figure 2a). They consist of separated fibers of the mineral with numerous inclusions of natrolite, albite, aegirine, parakeldyshite, lorenzenite, and fluorcalciopyrochlore between them (Figure 2b).
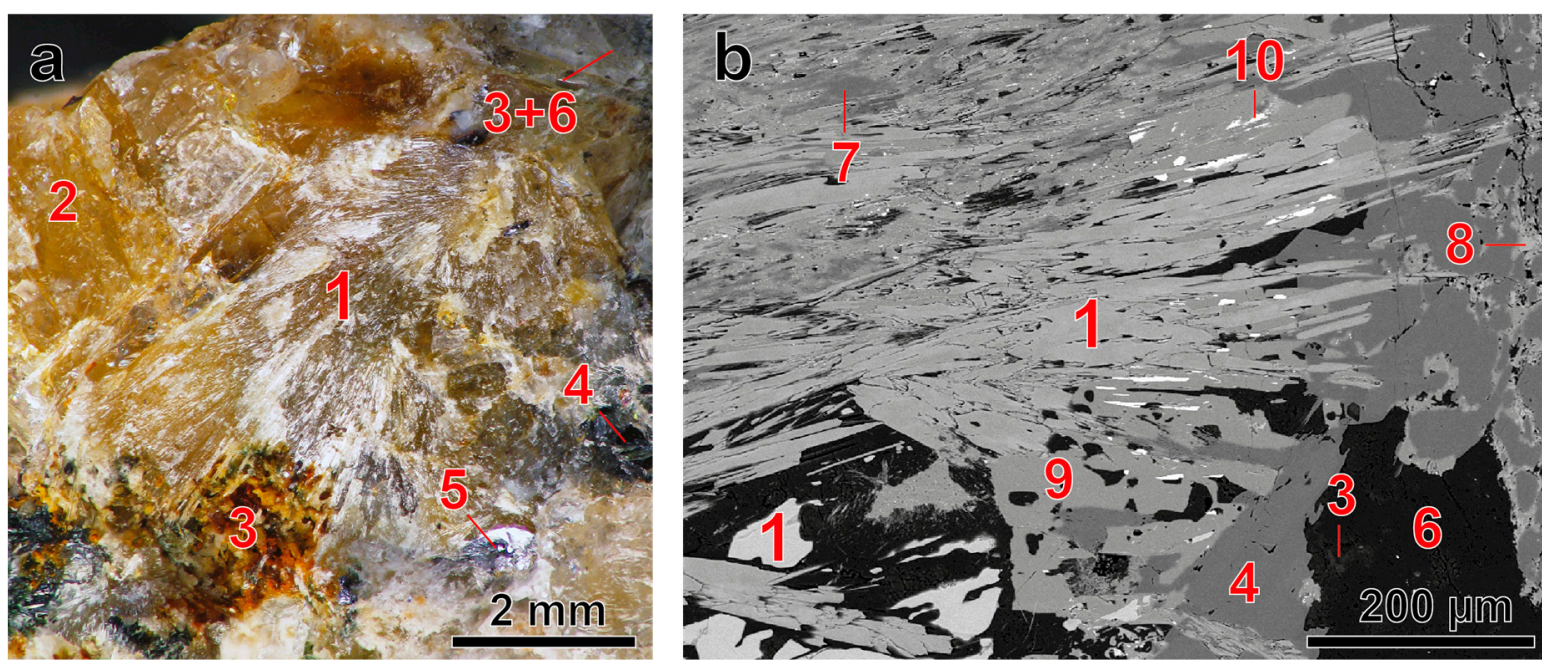

Figure 2. Radiated aggregate of chirvinskyite (1) in apatite segregation from albitized and natrolitized nepheline-microcline-aegirine-augite pegmatite in foyaite of Mt. Takhtarvumchorr (a) and BSE-image of such aggregate (b). 2-fluorapatite, 3-albite, 4-aegirine, 5-molybdenite, 6-natrolite, 7-lorenzenite, 8-eudialyte, 9-parakeldyshite, and 10-fluorcalciopyrochlore.

\subsection{Appearance and Physical Properties}

Chirvinskyite forms sheaf-like and radiated aggregates (up to $6 \mathrm{~mm}$ in diameter) of split fibrous metacrysts (up to $0.02 \mathrm{~mm}$ ) hosted in fine-grained natrolite, fluorapatite, and/or albite (Figure 2). No idiomorphic crystals have been found. The $a: b: c$ ratio calculated from the unit-cell parameters (single-crystal data) is close to 0.7:1:1.2. No twinning was observed. Cleavage and parting were not observed due to the splitting of chirvinskyite crystals into nanometer-sized fibers. The mineral is brittle and has a fibrous fracture. Mohs hardness is about 5 (hardly scratched with a steel needle). The density measured is $3.07(2) \mathrm{g} \cdot \mathrm{cm}^{-3}$, the structural density is $3.33 \mathrm{~g} \cdot \mathrm{cm}^{-3}$, and the density calculated using the empirical formula and single-crystal unit-cell parameters is $3.41 \mathrm{~g} \cdot \mathrm{cm}^{-3}$. The difference between calculated and observed values may be caused by numerous inclusions of surrounding minerals (mostly natrolite, albite, aegirine, parakeldyshite, and fluorapatite).

Macroscopically, chirvinskyite is pale creamy. Its luster is silky along the fibers, and dull in cross-sections. The mineral is transparent in separate fibers, translucent in aggregates, and has a white streak. It is biaxial, negative, $\alpha=1.670(2), \beta=1.690(2), \gamma=1.705(2)(589 \mathrm{~nm}), 2 V_{\text {calc }}=80.9^{\circ}$. The optical orientation is $Z=b, X^{\wedge} c=14^{\circ}$, and further details remain unclear. In transmitted light, chirvinskyite is 
colourless, without pleochroism or dispersion. The mineral is non-fluorescent. The Gladstone-Dale calculation provides a compatibility index of 0.028 , which is regarded as excellent.

\subsection{Chemical Composition}

Table 1 provides the mean analytical results for 17 chirvinskyite aggregates. Taking into account structural data, the average empirical formula of chirvinskyite (based on $\mathrm{Si}=8$ apfu) can be written as $\left(\mathrm{Na}_{9.81} \mathrm{Ca}_{3.28} \mathrm{~K}_{0.01}\right)_{\sum 13.10}\left(\mathrm{Fe}_{0.72} \mathrm{Mn}_{0.69} \square_{0.54} \mathrm{Mg}_{0.05}\right)_{\sum 2.00}\left(\mathrm{Ti}_{1.81} \mathrm{Nb}_{0.19}\right)_{\sum 2.00}\left(\mathrm{Zr}_{2.27} \mathrm{Ti}_{0.63}\right)_{\sum 2.90}\left(\mathrm{Si}_{2} \mathrm{O}_{7}\right)_{4}$ $\left\{(\mathrm{OH})_{5.94} \mathrm{O}_{3.09} \mathrm{~F}_{2.97}\right\}_{\Sigma 12.00}$. Taking into account the structural data, the simplified formula of the mineral is $(\mathrm{Na}, \mathrm{Ca})_{13}(\mathrm{Fe}, \mathrm{Mn}, \mathrm{\square})_{2}(\mathrm{Ti}, \mathrm{Nb})_{2}(\mathrm{Zr}, \mathrm{Ti})_{3}\left(\mathrm{Si}_{2} \mathrm{O}_{7}\right)_{4}(\mathrm{OH}, \mathrm{O}, \mathrm{F})_{12}$, or $A_{13} B_{2} C_{2} D_{3}\left(T_{2} \mathrm{O}_{7}\right)_{4} X_{12}$, where $A=\mathrm{Na}$, $\mathrm{Ca}, \mathrm{K}, B=\mathrm{Fe}, \mathrm{Mn}, \mathrm{Mg}$, vacancy, $\mathrm{C}=\mathrm{Ti}, \mathrm{Nb}, \mathrm{D}=\mathrm{Zr}, \mathrm{Ti}, \mathrm{T}=\mathrm{Si}, X=\mathrm{O}, \mathrm{OH}, \mathrm{F}$. The formula of a theoretical end member is $\left(\mathrm{Na}_{8} \mathrm{Ca}_{5}\right) \mathrm{Fe}^{2+}{ }_{2} \mathrm{Ti}_{2} \mathrm{Zr}_{3}\left(\mathrm{Si}_{2} \mathrm{O}_{7}\right)_{4} \mathrm{O}_{6}(\mathrm{OH})_{6}$.

Table 1. Chemical composition of chirvinskyite.

\begin{tabular}{cccccc}
\hline Sample & Mean & SD & Min & Max & $n$ \\
\hline $\mathrm{H}_{2} \mathrm{O}^{*}, \mathrm{wt} \%$ & 3.14 & & & & 1 \\
$\mathrm{~F}$ & 3.32 & 0.20 & 3.12 & 3.52 & 3 \\
$\mathrm{Na}_{2} \mathrm{O}$ & 17.85 & 1.04 & 16.90 & 20.59 & 17 \\
$\mathrm{MgO}$ & 0.13 & 0.09 & 0.00 & 0.29 & 17 \\
$\mathrm{SiO}$ & 28.22 & 0.49 & 27.76 & 29.44 & 17 \\
$\mathrm{~K}_{2} \mathrm{O}$ & 0.03 & 0.02 & 0.00 & 0.05 & 17 \\
$\mathrm{CaO}$ & 10.80 & 0.34 & 10.21 & 11.36 & 17 \\
$\mathrm{TiO}$ & 11.46 & 0.98 & 9.79 & 12.80 & 17 \\
$\mathrm{MnO}$ & 2.87 & 0.50 & 2.17 & 3.89 & 17 \\
$\mathrm{FeO}$ & 3.03 & 0.39 & 2.32 & 3.99 & 17 \\
$\mathrm{ZrO}$ & 16.43 & 1.71 & 13.63 & 19.43 & 17 \\
$\mathrm{Nb}_{2} \mathrm{O}_{5}$ & 1.46 & 0.35 & 0.88 & 2.06 & 17 \\
$\mathrm{~F}=\mathrm{O}$ & -1.40 & & & & \\
$\mathrm{Total}$ & 97.34 & & & &
\end{tabular}

* Water content calculated from structural data $\left(\mathrm{H}_{2} \mathrm{O}\right.$ content determined by the Penfield method is about $\left.7 \mathrm{wt} \%\right)$.

Despite the fact that iron predominates in the $B$-position of chirvinskyite, manganese is its significant constituent as well. The total content of Fe, Mn, and $\mathrm{Mg}$ in chirvinskyite linearly decreases with the increase of the Ca content (Figure 3a). The Zr content decreases with the increase of the Ti content from $\mathrm{Zr}_{3} \mathrm{Ti}_{2}$ to $\mathrm{Zr}_{2} \mathrm{Ti}_{3}$ (Figure $3 b$ ). Therefore, the main isomorphic substitutions in chirvinskyite can be written as the following formula shown below.

$$
\begin{gathered}
\mathrm{Fe}^{2+}{ }_{B} \rightarrow \mathrm{Mn}^{2+}{ }_{B} \\
2 \mathrm{Na}^{+}{ }_{A}+(\mathrm{Fe}, \mathrm{Mn}, \mathrm{Mg})^{2+}{ }_{B} \rightarrow 2 \mathrm{Ca}^{2+}{ }_{A}+\square_{B} ; \\
\mathrm{Zr}^{4+}{ }_{D} \rightarrow \mathrm{Ti}^{4+}{ }_{D} .
\end{gathered}
$$

In limiting cases, these substitutions can lead to the formation of the following compounds, correspondingly.

$$
\begin{gathered}
\left(\mathrm{Na}_{8} \mathrm{Ca}_{5}\right) \mathrm{Mn}_{2} \mathrm{Ti}_{2} \mathrm{Zr}_{3}\left(\mathrm{Si}_{2} \mathrm{O}_{7}\right)_{4} \mathrm{O}_{6}(\mathrm{OH})_{6} \\
\left(\mathrm{Na}_{8} \mathrm{Ca}_{5}\right) \square_{2} \mathrm{Ti}_{2} \mathrm{Zr}_{3}\left(\mathrm{Si}_{2} \mathrm{O}_{7}\right)_{4} \mathrm{O}_{6}(\mathrm{OH})_{6} \\
\left(\mathrm{Na}_{8} \mathrm{Ca}_{5}\right) \mathrm{Fe}_{2} \mathrm{Ti}_{5}\left(\mathrm{Si}_{2} \mathrm{O}_{7}\right)_{4} \mathrm{O}_{6}(\mathrm{OH})_{6}
\end{gathered}
$$

Chirvinskyite does not dissolve in cold hydrochloric acid. 

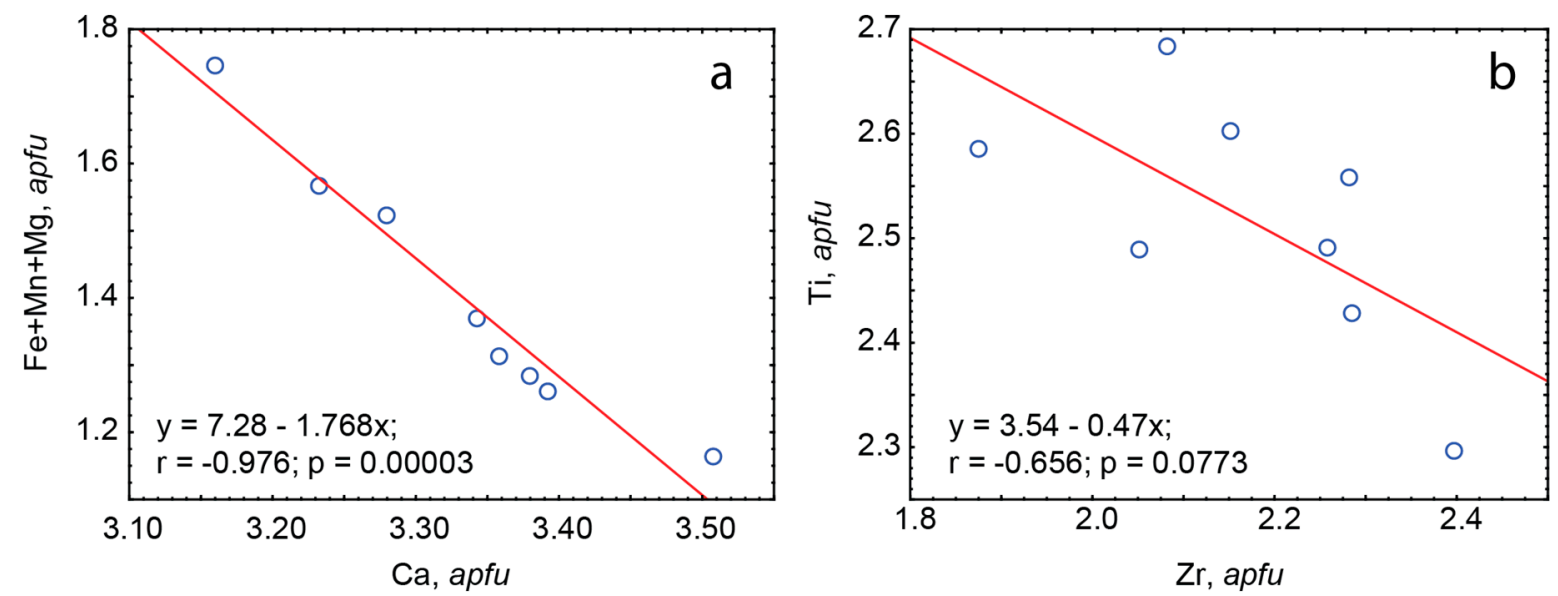

Figure 3. The relations between the cation contents in the $B(\mathbf{a})$ and $C-D(\mathbf{b})$ sites in chirvinskyite.

\subsection{Raman Spectroscopy}

The Raman spectrum of chirvinskyite (Figure 4) contains characteristic bands of the O-H stretching vibrations (Table 2) in the range from 2900 to $3600 \mathrm{~cm}^{-1}$. The intensive bands at 607,636s, and 652 as well as $330,347 \mathrm{w}, 413 \mathrm{w}$, and $445 \mathrm{~cm}^{-1}$ can be assigned to the $\mathrm{Si}-\mathrm{O}-\mathrm{Si}$ and $\mathrm{O}-\mathrm{Si}-\mathrm{O}$ asymmetric and symmetric modes of bending vibrations in the $\left[\mathrm{Si}_{2} \mathrm{O}_{7}\right]$-groups. The bands observed in the ranges of 900 to 1000 and 700 to $850 \mathrm{~cm}^{-1}$ can be related to the asymmetric and symmetric vibrations of the same bonds [20,21]. The bands at 481s, 497, 538,593,177, and $196 \mathrm{~cm}^{-1}$ are assigned to the bending and stretching vibrations of the Ti-O, $\mathrm{Zr}-\mathrm{O}, \mathrm{Mn}-\mathrm{O}$, and Fe-O bonds in respective cation-centered octahedra [22,23]. The bands at the 233, 256s, 272, 289, and $303 \mathrm{~cm}^{-1}$ correspond to the bending/stretching vibrations of the Ca-O and $\mathrm{Na}-\mathrm{O}$ bonds of the $\mathrm{Ca}(\mathrm{Na}) \mathrm{O}_{8}$ and $\mathrm{Ca}(\mathrm{Na}) \mathrm{O}_{7}$ coordination polyhedra. The bands below $150 \mathrm{~cm}^{-1}$ can be assigned to the lattice vibrations. The absence of absorption bands in the range of 1100 to $1650 \mathrm{~cm}^{-1}$ indicates the absence of boron-bearing groups and water molecules. The $d_{\text {calc }}(\mathrm{O} \cdots \mathrm{O})$ distances calculated according to the Libowitzky [24] formula for the peaks at 3554, 3504, 3073, and $2918 \mathrm{~cm}^{-1}$ are equal to $3.32,3.21,2.97$, and $2.94(\AA)$. The band at $3554 \mathrm{~cm}^{-1}\left(d_{\text {calc }}=3.32 \AA\right)$ corresponds to the $\mathrm{X}(18) \cdots \mathrm{O}(15)$ bond. The respective $\mathrm{O} \cdots \mathrm{O}$ distance in the structure is $3.282 \AA$. The band at $3504 \mathrm{~cm}^{-1}\left(d_{\text {calc }}=3.21 \AA\right)$ may be associated with the following $\mathrm{O} \cdots$ O distances corresponding to weak hydrogen bonds: $3.205 \AA$ for $(\mathrm{X}(18) \cdots \mathrm{O}(8)$ and $3.206 \AA$ for $\mathrm{X}(19) \cdots \mathrm{O}(12)$, respectively. The bands at 3073 and $2918 \mathrm{~cm}^{-1}$ with $d_{\text {calc }}=2.97$ and $2.94 \AA$ may be related to short contacts $X(16) \cdots O(12)$ and $\mathrm{X}(16) \cdots \mathrm{O}(8)$, with corresponding distances in the structure of 2.710 and $2.736 \AA$. These two bands may also be associated with an organic impurity.

Table 2. Raman bands in the chirvinskyite spectrum and their interpretation.

\begin{tabular}{ccc}
\hline Raman Shift, $\mathbf{c m}^{\mathbf{- 1}}$ & Assignment & Type \\
\hline $2918,3073,3054 \mathrm{sh}, 3554$ & $\mathrm{OH}$ & $v_{1}$ \\
$704 \mathrm{~s}, 729 \mathrm{sh}, 802,848$ & $\mathrm{Si}_{2} \mathrm{O}_{7}$ & $v_{1}$ \\
$898,924 \mathrm{sh}, 960,997$ & $\mathrm{Si}_{2} \mathrm{O}_{7}$ & $v_{3}$ \\
$330,347 \mathrm{w}, 413 \mathrm{w}, 445$ & $\mathrm{Si}_{2} \mathrm{O}_{7}$ & $v_{2}$ \\
$607,636 \mathrm{~s}, 652$ & $\mathrm{Si}_{2} \mathrm{O}_{7}$ & $v_{4}$ \\
$481 \mathrm{~s}, 497,538,593$ & $\mathrm{MeO}_{6}$ & $v_{1}, v_{2}$ \\
177,196 & $\mathrm{MeO}_{6}$ & $v_{3}, v_{4}$ \\
$233,256 \mathrm{~s}, 272,289,303$ & $\mathrm{MeO}_{7}, \mathrm{MeO}_{8}$ & $v_{1}, v_{2}$ \\
$91 \mathrm{~s}, 123,145$ & & lattice vibrations \\
\hline
\end{tabular}

$\mathrm{sh}=$ shoulder. $\mathrm{s}=$ strong intensity. $\mathrm{w}=$ weak. 


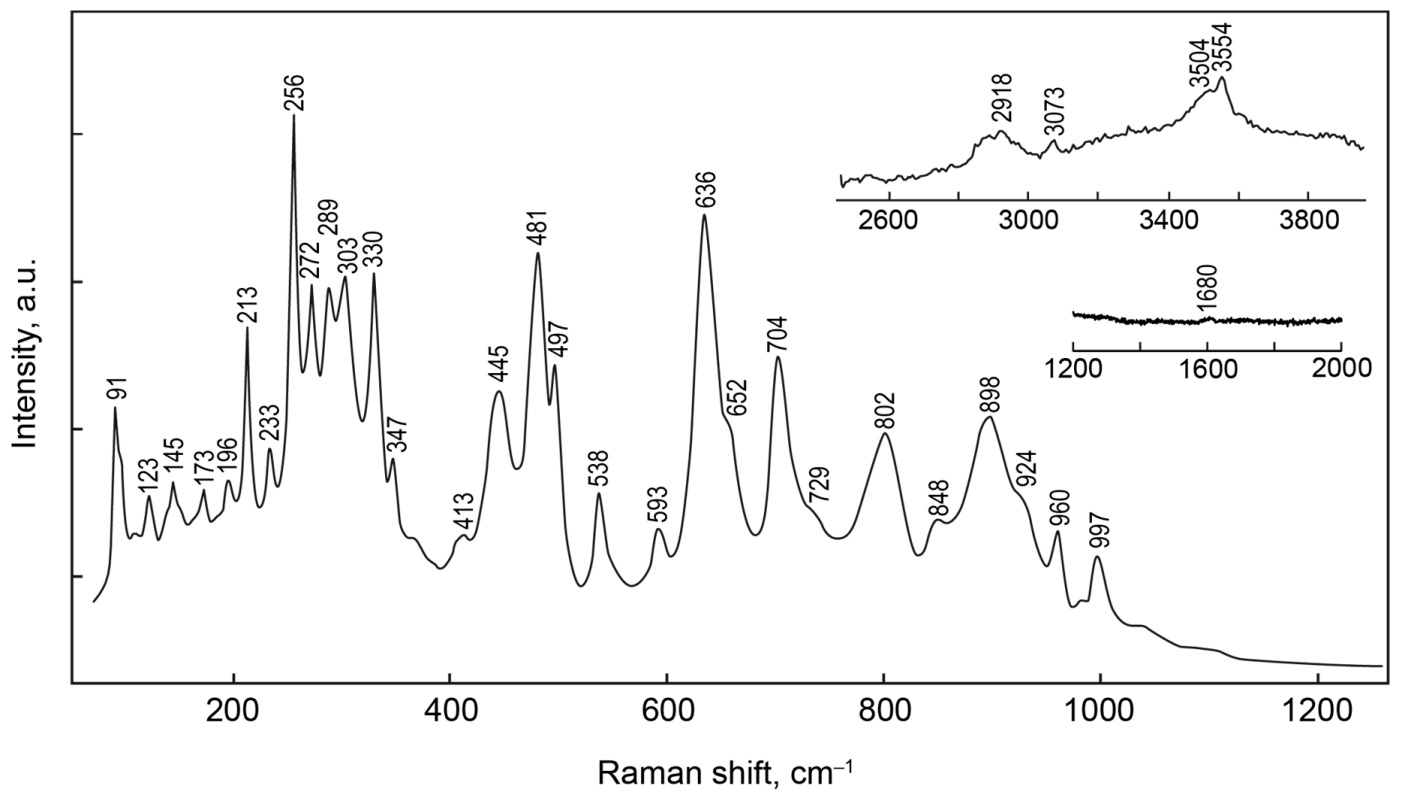

Figure 4. Raman spectrum of chirvinskyite. The spectrum intensity at $2600-3900$ and $1200-2000 \mathrm{~cm}^{-1}$ is enlarged by two.

\subsection{Powder X-ray Diffraction}

The unit-cell parameters of chirvinskyite determined from the powder pattern (Table 3) are: 7.0485(6), $b=9.8557(8), c=12.2233(8) \AA, \alpha=77.95(1), \beta=82.05(1), \gamma=90.03(1)^{\circ}, V=822.1(1) \AA^{3}, Z=1$, which are in good agreement with the single-crystal data. The strongest $X$-ray powder-diffraction lines (listed as $d$ in $\AA(I)(h k l))$ are as follows: 7.00(34)(100), 5.907(17)(002), 3.956(23)(013), 3.416(33)(023), 2.886(57)(132), 2.796(100)(024), 1.7407(25)(402), and 1.6461(16)(037).

Table 3. X-ray powder-diffraction data for chirvinskyite.

\begin{tabular}{|c|c|c|c|c|c|c|c|}
\hline$I_{\text {meas }}$ & $d_{\text {meas }}, \AA$ & $d_{\text {calc }}, \AA$ & $h k l$ & $I_{\text {meas }}$ & $d_{\text {meas, }} \AA$ & $d_{\text {calc }, ~} \AA$ & $h k l$ \\
\hline 5 & 11.86 & 11.83 & 001 & 2 & 2.382 & 2.381 & 134 \\
\hline 5 & 9.67 & 9.63 & 010 & 12 & 2.326 & 2.327 & 025 \\
\hline 2 & 8.40 & 8.39 & 011 & 2 & 2.276 & 2.277 & 302 \\
\hline 34 & 7.00 & 6.98 & 100 & 9 & 2.193 & 2.195 & -115 \\
\hline 2 & 6.41 & 6.42 & 101 & 4 & 2.131 & 2.132 & 035 \\
\hline 17 & 5.907 & 5.916 & 002 & 5 & 2.109 & 2.107 & -233 \\
\hline 1 & 5.197 & 5.179 & -111 & 9 & 2.018 & 2.018 & 016 \\
\hline 9 & 4.844 & 4.832 & 021 & 2 & 2.007 & 2.004 & $-2-32$ \\
\hline 6 & 4.632 & 4.638 & $-1-11$ & 3 & 1.9878 & 1.9846 & $2-15$ \\
\hline 3 & 4.222 & 4.228 & -102 & 8 & 1.9677 & 1.9664 & $0-25$ \\
\hline 5 & 4.139 & 4.137 & -112 & 3 & 1.9275 & 1.9268 & 050 \\
\hline 15 & 4.039 & 4.024 & 121 & 3 & 1.9029 & 1.8985 & -151 \\
\hline 23 & 3.956 & 3.955 & 013 & 11 & 1.8610 & 1.8617 & 216 \\
\hline 1 & 3.724 & 3.718 & 122 & 7 & 1.7670 & 1.7651 & 154 \\
\hline 5 & 3.656 & 3.651 & $-1-12$ & 6 & 1.7528 & 1.7514 & $-2-15$ \\
\hline 2 & 3.489 & 3.489 & 200 & 25 & 1.7407 & 1.7413 & 402 \\
\hline 33 & 3.416 & 3.427 & 023 & 2 & 1.6927 & 1.6927 & -341 \\
\hline 7 & 3.245 & 3.243 & -103 & 1 & 1.6814 & 1.6833 & -411 \\
\hline 3 & 3.212 & 3.210 & 202 & 1 & 1.6722 & 1.6712 & $3-41$ \\
\hline 2 & 3.143 & 3.140 & -211 & 16 & 1.6461 & 1.6458 & 037 \\
\hline 11 & 3.012 & 3.012 & 014 & 2 & 1.6260 & 1.6262 & -146 \\
\hline 6 & 2.985 & 2.982 & $-2-11$ & 4 & 1.6117 & 1.6144 & $14-4$ \\
\hline
\end{tabular}


Table 3. Cont.

\begin{tabular}{cccccccc}
\hline $\boldsymbol{I}_{\text {meas }}$ & $\boldsymbol{d}_{\text {meas }}, \AA$ & $\boldsymbol{d}_{\text {calc } \boldsymbol{\prime}}, \AA$ & $\boldsymbol{h} \boldsymbol{k} \boldsymbol{l}$ & $\boldsymbol{I}_{\text {meas }}$ & $\boldsymbol{d}_{\text {meas }}, \AA$ & $\boldsymbol{d}_{\text {calc } \boldsymbol{\prime}}, \AA$ & $\boldsymbol{h} \boldsymbol{k} \boldsymbol{l}$ \\
\hline 4 & 2.932 & 2.941 & $-1-22$ & 7 & 1.6041 & 1.6057 & 060 \\
$\mathbf{5 7}$ & $\mathbf{2 . 8 8 6}$ & $\mathbf{2 . 8 9 0}$ & $\mathbf{1 3 2}$ & 6 & 1.5724 & 1.5727 & -315 \\
$\mathbf{1 0 0}$ & $\mathbf{2 . 7 9 6}$ & $\mathbf{2 . 7 9 8}$ & $\mathbf{0 2 4}$ & 6 & 1.5536 & 1.5528 & $30-5$ \\
3 & 2.706 & 2.708 & 124 & 4 & 1.5475 & 1.5461 & $33-3$ \\
2 & 2.637 & 2.632 & $-2-12$ & 6 & 1.4943 & 1.4961 & $-3-42$ \\
7 & 2.614 & 2.618 & $2-13$ & 13 & 1.4854 & 1.4849 & $4-24$ \\
4 & 2.588 & 2.589 & -222 & 7 & 1.4677 & 1.4676 & $4-15$ \\
2 & 2.522 & 2.531 & -133 & 1 & 1.4382 & 1.4368 & $-20-8$ \\
12 & 2.451 & 2.448 & 034 & 1 & 1.4182 & 1.4187 & $-31-7$ \\
9 & 2.413 & 2.415 & 042 & 6 & 1.3974 & 1.3982 & $4-25$ \\
\hline
\end{tabular}

The strongest $X$-ray powder-diffraction lines are given in bold.

\subsection{Crystal Structure}

Crystal data, data-collection information, and structure-refinement details are given in Table 4, atom coordinates, occupancies, bond valence sums, and displacement parameters are given in Tables 5 and 6, and selected interatomic distances are given in Table 7.

Table 4. Data and structure refinement parameters for chirvinskyite.

\begin{tabular}{|c|c|}
\hline Crystal system & Triclinic \\
\hline Space group & $P-1$ \\
\hline$a, \AA$ & $7.0477(5)$ \\
\hline$b, \AA$ & $9.8725(5)$ \\
\hline$c, \AA$ & $12.2204(9)$ \\
\hline$\alpha,{ }^{\circ}$ & $77.995(5)$ \\
\hline$\beta, \circ$ & $82.057(6)$ \\
\hline$\gamma, \circ$ & $89.988(5)$ \\
\hline Volume, $\AA^{3}$ & $823.35(9)$ \\
\hline Z & 1 \\
\hline$D_{\text {calc }}, \mathrm{g} / \mathrm{cm}^{3}$ & 3.332 \\
\hline$\mu, \mathrm{mm}^{1}$ & 3.260 \\
\hline $\mathrm{F}(000)$ & 802.0 \\
\hline Radiation & $\operatorname{MoK} \alpha(\lambda=0.71073)$ \\
\hline $2 \Theta$ range for data collection, ${ }^{\circ}$ & 3.506 to 75.382 \\
\hline Index ranges & $-9 \leq \mathrm{h} \leq 9,-13 \leq \mathrm{k} \leq 13,-11 \leq 1 \leq 16$ \\
\hline Reflections collected & 11456 \\
\hline Independent reflections & $4394\left[R_{\mathrm{int}}=0.0384, R_{\mathrm{sigma}}=0.0345\right]$ \\
\hline Data/restraints/parameters & $4394 / 0 / 319$ \\
\hline GooF & 1.111 \\
\hline Final $R$ indexes $[I>=2 \sigma(\mathrm{I})]$ & $R_{1}=0.0736, \mathrm{w} R_{2}=0.2016$ \\
\hline Final $R$ indexes [all data] & $R_{1}=0.0813, \mathrm{w} R_{2}=0.2067$ \\
\hline Largest difference peak/hole/e $\AA^{-3}$ & $2.23 /-1.47$ \\
\hline
\end{tabular}

Table 5. Atomic coordinates, occupancies, bond valence sums, experimental and calculated site-scattering factors ( $\mathrm{SSF}_{\exp }$ and $\mathrm{SSF}_{\text {calc, }}$, respectively), and equivalent isotropic displacement parameters $\left(\AA^{2} \times 10^{-3}\right)$ of chirvinskyite.

\begin{tabular}{ccccccccc}
\hline Site & $x$ & $y$ & $z$ & Occupancy & SSF $_{\text {exp }}\left(e^{-}\right)$ & SSF $_{\text {calc }}\left(e^{-}\right)$ & BVS $^{*}$ & $U^{\text {iso }}$ \\
\hline$A 1$ & $-0.0800(2)$ & $0.9365(1)$ & $0.3232(1)$ & $\mathrm{Ca}$ & 20.00 & 20.00 & 2.37 & $13.7(3)$ \\
$A 2$ & $-0.0613(3)$ & $0.4585(2)$ & $0.2439(2)$ & $\mathrm{Ca}_{0.59} \mathrm{Na}_{0.41}$ & 16.31 & 16.31 & $1.96(2.35)$ & $16.0(7)$ \\
$A 3$ & $-0.1393(4)$ & $0.3604(2)$ & $0.5589(2)$ & $\mathrm{Na}_{0.78} \mathrm{Ca}_{0.22}$ & 12.98 & 12.98 & $1.46(1.32)$ & $17.3(10)$ \\
$A 4$ & $-0.2550(4)$ & $0.5007(3)$ & $-0.0016(2)$ & $\mathrm{Na}$ & 11.00 & 11.00 & 1.32 & $19.9(7)$ \\
\hline
\end{tabular}


Table 5. Cont.

\begin{tabular}{|c|c|c|c|c|c|c|c|c|}
\hline Site & $x$ & $y$ & $z$ & Occupancy & $\operatorname{SSF}_{\exp }\left(e^{-}\right)$ & $\operatorname{SSF}_{\text {calc }}\left(e^{-}\right)$ & BVS * & $U^{\text {iso }}$ \\
\hline$A 5$ & $-0.2851(3)$ & $0.2105(2)$ & $0.1348(2)$ & $\mathrm{Na}_{0.77} \mathrm{Ca}_{0.23}$ & 14.27 & 14.27 & $1.51(1.36)$ & $14.6(10)$ \\
\hline$A 6$ & $0.2193(4)$ & $0.2111(3)$ & $0.1328(2)$ & $\mathrm{Na}_{0.90} \mathrm{Ca}_{0.10}$ & 11.90 & 11.90 & $1.38(1.32)$ & $15.2(11)$ \\
\hline$A 7$ & 0 & 0 & 0 & $\mathrm{Ca}_{0.54} \mathrm{Na}_{0.46}$ & 15.86 & 15.86 & $1.82(2.24)$ & $18.3(11)$ \\
\hline$B^{* *}$ & $-0.3655(2)$ & $0.6437(1)$ & $0.4443(1)$ & $\begin{array}{c}\mathrm{Fe}_{0.36} \mathrm{Mn}_{0.345} \\
\square_{0.27} \mathrm{Mg}_{0.025}\end{array}$ & 20.80 & 18.29 & 2.17 & $11.1(4)$ \\
\hline C & $0.5607(2)$ & $0.5380(1)$ & $0.74501(9)$ & $\mathrm{Ti}_{0.94} \mathrm{Nb}_{0.06}$ & 23.14 & 23.14 & $3.76(3.71)$ & $8.9(4)$ \\
\hline$D 1$ & 0.5 & 0 & 0 & $\mathrm{Zr}_{0.53} \mathrm{Ti}_{0.47}$ & 31.54 & 31.54 & $3.58(4.17)$ & $8.1(4)$ \\
\hline D2 & $0.5777(1)$ & $0.06435(8)$ & $0.68359(6)$ & $\mathrm{Zr}_{0.58} \mathrm{Ti}_{0.42}$ & 32.44 & 32.44 & $3.59(4.12)$ & $8.2(3)$ \\
\hline$T 1$ & $0.2745(3)$ & $0.2522(2)$ & $0.8359(2)$ & $\mathrm{Si}$ & 14.00 & 14.00 & 4.09 & $8.9(4)$ \\
\hline$T 2$ & $0.8063(3)$ & $0.2503(2)$ & $0.8348(2)$ & $\mathrm{Si}$ & 14.00 & 14.00 & 4.10 & $8.4(4)$ \\
\hline T3 & $0.3281(3)$ & $0.8055(2)$ & $0.6049(2)$ & $\mathrm{Si}$ & 14.00 & 14.00 & 4.15 & 12.5(4) \\
\hline T4 & $-0.1382(3)$ & $0.8016(2)$ & $0.6059(2)$ & $\mathrm{Si}$ & 14.00 & 14.00 & 4.15 & $9.2(4)$ \\
\hline $\mathrm{O} 1$ & $0.3024(9)$ & $0.1492(6)$ & $0.9513(4)$ & $\mathrm{O}$ & 8.00 & 8.00 & $2.04(2.17)$ & $19.3(11)$ \\
\hline $\mathrm{O} 2$ & $0.3360(9)$ & $0.4102(6)$ & $0.8257(5)$ & $\mathrm{O}$ & 8.00 & 8.00 & $2.02(2.05)$ & $23.1(12)$ \\
\hline $\mathrm{O} 3$ & $0.3512(9)$ & $0.1947(5)$ & $0.7235(4)$ & $\mathrm{O}$ & 8.00 & 8.00 & $2.00(2.06)$ & $19.0(11)$ \\
\hline $\mathrm{O} 4$ & $0.039(1)$ & $0.2577(7)$ & $0.8365(6)$ & $\mathrm{O}$ & 8.00 & 8.00 & $2.08(2.11)$ & $32.5(14)$ \\
\hline O5 & $0.7206(8)$ & $0.1492(6)$ & $0.9520(4)$ & $\mathrm{O}$ & 8.00 & 8.00 & $2.01(2.13)$ & $18.5(11)$ \\
\hline O6 & $0.7874(8)$ & $0.1889(5)$ & $0.7244(4)$ & $\mathrm{O}$ & 8.00 & 8.00 & $2.10(2.17)$ & $17.0(11)$ \\
\hline O7 & $0.7468(9)$ & $0.4085(6)$ & $0.8230(5)$ & $\mathrm{O}$ & 8.00 & 8.00 & $1.98(2.01)$ & $23.6(12)$ \\
\hline O8 & $0.3643(9)$ & $0.6787(5)$ & $0.7045(5)$ & $\mathrm{O}$ & 8.00 & 8.00 & $2.10(2.11)$ & $22.0(12)$ \\
\hline O9 & $0.3743(9)$ & $0.9497(6)$ & $0.6342(5)$ & $\mathrm{O}$ & 8.00 & 8.00 & $2.11(2.21)$ & $22.9(12)$ \\
\hline $\mathrm{O} 10$ & $0.3976(8)$ & $0.7876(5)$ & $0.4788(4)$ & $\mathrm{O}$ & 8.00 & 8.00 & $1.96(1.99)$ & $15.4(10)$ \\
\hline $\mathrm{O} 11$ & $0.0896(9)$ & $0.8051(8)$ & $0.6215(6)$ & $\mathrm{O}$ & 8.00 & 8.00 & $2.10(2.11)$ & $35.1(15)$ \\
\hline $\mathrm{O} 12$ & $-0.2231(9)$ & $0.6766(6)$ & $0.7078(5)$ & $\mathrm{O}$ & 8.00 & 8.00 & $2.08(2.10)$ & $23.2(12)$ \\
\hline $\mathrm{O} 13$ & $-0.200(1)$ & $0.9498(6)$ & $0.6309(5)$ & $\mathrm{O}$ & 8.00 & 8.00 & $2.01(2.10)$ & $26.0(13)$ \\
\hline $\mathrm{O} 14$ & $-0.149(1)$ & $0.7765(6)$ & $0.4828(5)$ & $\mathrm{O}$ & 8.00 & 8.00 & $2.10(2.05)$ & $25.0(13)$ \\
\hline$X 15$ & $0.5817(8)$ & $0.4853(6)$ & $0.6104(4)$ & $\mathrm{O}_{0.70} \mathrm{~F}_{0.30}$ & 8.00 & 8.30 & $1.76(1.68)$ & 19.2(11) \\
\hline$X 16$ & $0.5228(6)$ & $0.6215(4)$ & $0.9006(4)$ & $(\mathrm{OH})$ & 8.00 & 8.00 & $1.32(1.28)$ & $6.2(8)$ \\
\hline$X 17$ & $0.5369(7)$ & $0.9556(5)$ & $0.8415(4)$ & $\mathrm{O}_{0.89} \mathrm{~F}_{0.11}$ & 8.00 & 8.11 & $1.83(2.03)$ & $15.8(10)$ \\
\hline$X 18$ & $-0.1189(8)$ & $0.5239(5)$ & $0.3995(4)$ & $(\mathrm{OH})_{0.90} \mathrm{O}_{0.10}$ & 8.00 & 8.00 & $1.48(1.51)$ & $14.3(10)$ \\
\hline X19 & $-0.0248(7)$ & $0.3710(4)$ & $0.0870(4)$ & $(\mathrm{OH})_{0.95} \mathrm{O}_{0.05}$ & 8.00 & 8.00 & 1.34 (1.39) & $9.4(9)$ \\
\hline$X 20$ & $-0.0394(6)$ & $0.0669(5)$ & $0.1592(4)$ & $\mathrm{O}_{0.70} \mathrm{~F}_{0.30}$ & 8.00 & 8.30 & $1.76(1.83)$ & $8.1(8)$ \\
\hline
\end{tabular}

Table 6. Anisotropic displacement parameters $\left(\AA^{2}\right)$ for chirvinskyite.

\begin{tabular}{ccccccc}
\hline Atom & $\boldsymbol{U}^{\mathbf{1 1}}$ & $\boldsymbol{U}^{\mathbf{2 2}}$ & $\boldsymbol{U}^{\mathbf{3 3}}$ & $\boldsymbol{U}^{\mathbf{2 3}}$ & $\boldsymbol{U}^{\mathbf{1 3}}$ & $\boldsymbol{U}^{\mathbf{1 2}}$ \\
\hline A1 & $0.0167(7)$ & $0.0087(6)$ & $0.0139(7)$ & $0.0010(5)$ & $-0.0015(5)$ & $0.0011(5)$ \\
A2 & $0.0204(11)$ & $0.0103(10)$ & $0.0175(11)$ & $-0.0016(7)$ & $-0.0052(7)$ & $0.0007(6)$ \\
A3 & $0.0229(16)$ & $0.0116(14)$ & $0.0149(14)$ & $0.0018(9)$ & $-0.0006(9)$ & $0.0022(9)$ \\
A4 & $0.0230(16)$ & $0.0207(16)$ & $0.0148(15)$ & $-0.0015(12)$ & $-0.0021(12)$ & $0.0005(13)$ \\
A5 & $0.0153(14)$ & $0.0126(13)$ & $0.0169(14)$ & $-0.0056(9)$ & $-0.0022(9)$ & $0.0005(8)$ \\
A6 & $0.0178(17)$ & $0.0125(15)$ & $0.0173(16)$ & $-0.0072(10)$ & $-0.0031(10)$ & $0.0018(10)$ \\
A7 & $0.0240(17)$ & $0.0151(15)$ & $0.0150(15)$ & $-0.002(1)$ & $-0.0025(10)$ & $0.0024(10)$ \\
B & $0.0137(7)$ & $0.0088(6)$ & $0.0107(6)$ & $-0.0009(4)$ & $-0.0028(4)$ & $-0.0004(4)$ \\
C & $0.0112(6)$ & $0.0048(5)$ & $0.0097(6)$ & $0.0007(4)$ & $-0.0017(4)$ & $0.0006(4)$ \\
D1 & $0.0091(6)$ & $0.0051(6)$ & $0.0093(6)$ & $0.0007(4)$ & $-0.0019(4)$ & $0.0003(4)$ \\
D2 & $0.0100(4)$ & $0.0050(4)$ & $0.0099(4)$ & $-0.0020(3)$ & $-0.0021(3)$ & $0.0004(3)$ \\
T1 & $0.0158(9)$ & $0.035(7)$ & $0.0069(8)$ & $0.0000(6)$ & $-0.0008(6)$ & $-0.0003(6)$ \\
T2 & $0.0137(8)$ & $0.046(7)$ & $0.0065(8)$ & $-0.0004(6)$ & $-0.0013(6)$ & $0.0009(6)$ \\
T3 & $0.023(1)$ & $0.056(8)$ & $0.0091(8)$ & $-0.0017(6)$ & $-0.0022(7)$ & $0.0011(7)$ \\
T4 & $0.0143(9)$ & $0.048(7)$ & $0.0090(8)$ & $-0.0018(6)$ & $-0.0030(6)$ & $0.0020(6)$ \\
O1 & $0.033(3)$ & $0.017(2)$ & $0.008(2)$ & $-0.0018(18)$ & $-0.001(2)$ & $0.004(2)$ \\
O2 & $0.038(3)$ & $0.014(2)$ & $0.017(3)$ & $-0.003(2)$ & $-0.005(2)$ & $-0.002(2)$ \\
O3 & $0.034(3)$ & $0.014(2)$ & $0.009(2)$ & $-0.0016(18)$ & $-0.001(2)$ & $0.002(2)$ \\
O4 & $0.027(3)$ & $0.037(4)$ & $0.035(3)$ & $-0.008(3)$ & $-0.008(3)$ & $0.001(3)$ \\
O5 & $0.029(3)$ & $0.019(3)$ & $0.007(2)$ & $-0.0016(18)$ & $0.0000(2)$ & $-0.003(2)$ \\
O6 & $0.029(3)$ & $0.011(2)$ & $0.011(2)$ & $-0.0034(18)$ & $-0.003(2)$ & $-0.002(2)$ \\
O7 & $0.036(3)$ & $0.014(2)$ & $0.020(3)$ & $-0.004(2)$ & $-0.003(2)$ & $0.002(2)$ \\
O8 & $0.042(4)$ & $0.009(2)$ & $0.015(2)$ & $-0.0014(19)$ & $-0.007(2)$ & $0.008(2)$ \\
\hline
\end{tabular}


Table 6. Cont.

\begin{tabular}{ccccccc}
\hline Atom & $\boldsymbol{U}^{\mathbf{1 1}}$ & $\boldsymbol{U}^{\mathbf{2 2}}$ & $\boldsymbol{U}^{\mathbf{3 3}}$ & $\boldsymbol{U}^{\mathbf{2 3}}$ & $\boldsymbol{U}^{\mathbf{1 3}}$ & $\boldsymbol{U}^{\mathbf{1 2}}$ \\
\hline O9 & $0.037(3)$ & $0.011(2)$ & $0.022(3)$ & $-0.006(2)$ & $-0.004(2)$ & $-0.004(2)$ \\
O10 & $0.025(3)$ & $0.013(2)$ & $0.007(2)$ & $-0.0023(17)$ & $0.0031(19)$ & $-0.0040(19)$ \\
O11 & $0.020(3)$ & $0.045(4)$ & $0.039(4)$ & $-0.008(3)$ & $-0.004(3)$ & $0.003(3)$ \\
O12 & $0.037(3)$ & $0.012(2)$ & $0.018(3)$ & $-0.001(2)$ & $0.0000(2)$ & $-0.005(2)$ \\
O13 & $0.042(4)$ & $0.014(3)$ & $0.024(3)$ & $-0.006(2)$ & $-0.008(3)$ & $0.005(2)$ \\
O14 & $0.045(4)$ & $0.019(3)$ & $0.012(2)$ & $-0.005(2)$ & $-0.006(2)$ & $0.001(2)$ \\
X15 & $0.026(3)$ & $0.017(3)$ & $0.013(2)$ & $-0.0026(19)$ & $0.001(2)$ & $0.005(2)$ \\
X16 & $0.009(2)$ & $0.0025(18)$ & $0.0059(19)$ & $0.0023(15)$ & $-0.0006(15)$ & $-0.0009(15)$ \\
X17 & $0.012(2)$ & $0.015(2)$ & $0.019(2)$ & $0.0006(19)$ & $-0.0041(19)$ & $-0.0011(18)$ \\
X18 & $0.025(3)$ & $0.010(2)$ & $0.008(2)$ & $-0.0024(17)$ & $-0.0007(18)$ & $-0.0008(19)$ \\
X19 & $0.014(2)$ & $0.0021(19)$ & $0.011(2)$ & $0.0018(16)$ & $-0.0028(17)$ & $-0.0007(16)$ \\
X20 & $0.008(2)$ & $0.008(2)$ & $0.007(2)$ & $0.0023(16)$ & $-0.0015(16)$ & $-0.0007(16)$ \\
\hline
\end{tabular}

Table 7. Selected interatomic distances (Å) for the crystal structure of chirvinskyite.

\begin{tabular}{|c|c|c|c|c|c|c|c|c|}
\hline$B$ & $\mathrm{O}^{4}$ & $2.310(5)$ & $T 2$ & $\mathrm{O} 4$ & $1.643(7)$ & $A 3$ & $06^{5}$ & $2.348(6)$ \\
\hline$B$ & $\mathrm{O} 10^{5}$ & $2.245(6)$ & $T 2$ & O5 & $1.606(5)$ & $A 3$ & $\mathrm{O} 10^{2}$ & $2.484(6)$ \\
\hline$B$ & O14 & $2.174(6)$ & $T 2$ & O6 & $1.612(5)$ & $A 3$ & $\mathrm{O} 11^{2}$ & $2.990(8)$ \\
\hline$B$ & $X 15^{2}$ & $2.242(6)$ & $T 2$ & O7 & $1.599(6)$ & $A 3$ & $\mathrm{O} 14^{2}$ & $2.493(7)$ \\
\hline$B$ & $X 15^{5}$ & $2.275(5)$ & $<\mathrm{T} 2-\mathrm{O}>$ & 1.615 & $A 3$ & $X 15^{5}$ & $2.400(6)$ & \\
\hline \multirow[t]{3}{*}{$B$} & X18 & $2.173(5)$ & & & & $A 3$ & X18 & $2.243(5)$ \\
\hline & $<B 1-\phi^{*}>$ & 2.237 & T3 & O8 & $1.602(6)$ & $A 3$ & $X 18^{2}$ & $2.318(6)$ \\
\hline & & & T3 & O9 & $1.583(5)$ & & $<A 3-\phi>$ & 2.468 \\
\hline$C$ & $\mathrm{O} 2^{5}$ & $2.031(6)$ & T3 & O10 & $1.597(5)$ & & & \\
\hline$C$ & O7 & $2.025(6)$ & T3 & O11 & $1.665(7)$ & $A 4$ & $\mathrm{O} 2^{4}$ & $2.484(6)$ \\
\hline$C$ & O8 & $1.998(6)$ & & $<\mathrm{T} 3-\mathrm{O}>$ & 1.612 & $A 4$ & $\mathrm{O}^{21}$ & $2.496(6)$ \\
\hline$C$ & $\mathrm{O} 12^{11}$ & $1.988(6)$ & & & & $A 4$ & $X^{2}$ & $2.298(5)$ \\
\hline$C$ & X15 & $1.813(5)$ & T4 & O11 & $1.644(7)$ & $A 4$ & $X^{21}$ & $2.286(5)$ \\
\hline \multirow[t]{3}{*}{$C$} & $X 16$ & $2.209(4)$ & T4 & O12 & $1.607(6)$ & $A 4$ & X19 & $2.313(6)$ \\
\hline & $<C 1-\phi>$ & 2.011 & T4 & O13 & $1.604(6)$ & $A 4$ & $\mathrm{X} 19^{20}$ & $2.338(5)$ \\
\hline & & & T4 & O14 & $1.587(6)$ & & $<A 4-\phi>$ & 2.369 \\
\hline$D 1$ & $\mathrm{O} 1^{5}$ & $2.080(6)$ & & $<\mathrm{T} 4-\mathrm{O}>$ & 1.611 & & & \\
\hline$D 1$ & $\mathrm{O} 1^{6}$ & $2.080(6)$ & & & & $A 5$ & $\mathrm{O} 5^{18}$ & $2.428(6)$ \\
\hline$D 1$ & $\mathrm{O}^{7}$ & $2.078(6)$ & $A 1$ & $\mathrm{O}^{4}$ & $2.503(6)$ & $A 5$ & $\mathrm{O} 8^{13}$ & $2.440(6)$ \\
\hline D1 & O5 & $2.078(6)$ & $A 1$ & $\mathrm{O} 4^{4}$ & $2.994(7)$ & $A 5$ & O913 & $2.919(6)$ \\
\hline$D 1$ & $X 17^{8}$ & $2.055(5)$ & $A 1$ & $\mathrm{O}^{4}$ & $2.468(6)$ & $A 5$ & $X 16^{13}$ & $2.376(5)$ \\
\hline \multirow[t]{3}{*}{$D 1$} & $X 17^{9}$ & $2.055(5)$ & $A 1$ & O913 & $2.404(6)$ & $A 5$ & $X 17^{13}$ & $2.363(6)$ \\
\hline & $<D 1-\phi>$ & 2.071 & $A 1$ & $\mathrm{O} 11^{13}$ & $2.770(8)$ & $A 5$ & $X 19^{16}$ & $2.353(5)$ \\
\hline & & & $A 1$ & $\mathrm{O} 13^{13}$ & $2.458(7)$ & $A 5$ & $X 20$ & $2.250(5)$ \\
\hline D2 & $\mathrm{O} 3^{15}$ & $2.118(6)$ & $A 1$ & O14 & $2.234(6)$ & & $<A 5-\phi>$ & 2.447 \\
\hline D2 & $\mathrm{O}^{16}$ & $2.098(5)$ & $A 1$ & $\mathrm{X} 20$ & $2.127(5)$ & & & \\
\hline D2 & O9 & $2.056(6)$ & & $<A 1-\phi>$ & 2.495 & A6 & $\mathrm{O} 1^{18}$ & $2.417(6)$ \\
\hline D2 & $\mathrm{O} 10^{12}$ & $2.192(5)$ & & & & A6 & $\mathrm{O} 12^{13}$ & $2.437(6)$ \\
\hline D2 & $\mathrm{O} 13^{11}$ & $2.041(6)$ & $A 2$ & $\mathrm{O} 2^{4}$ & $2.479(7)$ & A6 & $\mathrm{O} 13^{13}$ & 2.977(7) \\
\hline \multirow[t]{3}{*}{ D2 } & X17 & $1.986(5)$ & $A 2$ & $\mathrm{O} 4^{4}$ & $2.766(7)$ & A6 & $X 16^{12}$ & $2.391(5)$ \\
\hline & $<D 2-\phi>$ & 2.082 & $A 2$ & $\mathrm{O} 7^{4}$ & $2.522(7)$ & A6 & $X 17^{12}$ & $2.386(6)$ \\
\hline & & & $A 2$ & $\mathrm{O}^{2}$ & $2.463(7)$ & A6 & $X 19^{16}$ & $2.379(5)$ \\
\hline $\mathrm{T} 1$ & O1 & $1.593(5)$ & $A 2$ & $\mathrm{O} 11^{2}$ & $2.766(8)$ & $A 6$ & $X 20$ & $2.259(5)$ \\
\hline $\mathrm{T} 1$ & $\mathrm{O} 2$ & $1.594(6)$ & $A 2$ & $\mathrm{O} 12^{2}$ & $2.479(7)$ & & $<A 6-\phi>$ & 2.464 \\
\hline $\mathrm{T} 1$ & $\mathrm{O} 3$ & $1.619(5)$ & $A 2$ & $X 18$ & $2.120(5)$ & & & \\
\hline $\mathrm{T} 1$ & O4 & $1.662(7)$ & $A 2$ & X19 & $2.246(5)$ & $A 7$ & $\mathrm{O} 1^{18}$ & $2.521(6)$ \\
\hline
\end{tabular}


Table 7. Cont.

\begin{tabular}{|c|c|c|c|c|c|c|}
\hline \multirow[t]{8}{*}{$<\mathrm{T} 1-\mathrm{O}>$} & 1.617 & $<A 2-\phi>$ & 2.480 & $A 7$ & $\mathrm{O} 1^{4}$ & $2.521(6)$ \\
\hline & & & & $A 7$ & $\mathrm{O} 4^{18}$ & $2.876(7)$ \\
\hline & & & & $A 7$ & $\mathrm{O} 4^{4}$ & $2.876(7)$ \\
\hline & & & & $A 7$ & $\mathrm{O} 5^{18}$ & $2.520(6)$ \\
\hline & & & & $A 7$ & $\mathrm{O} 5^{4}$ & $2.520(6)$ \\
\hline & & & & $A 7$ & $X 20^{19}$ & $2.164(4)$ \\
\hline & & & & $A 7$ & $X 20$ & $2.164(4)$ \\
\hline & & & & & $<A 7-\phi>$ & 2.520 \\
\hline
\end{tabular}

The simplified formula of chirvinskyite is $A_{13} B_{2} C_{2} D_{3}\left(T_{2} \mathrm{O}_{7}\right)_{4} X_{12}$, where $A=\mathrm{Na}, \mathrm{Ca}, \mathrm{K}, B=\mathrm{Fe}$, $\mathrm{Mn}, \mathrm{Mg}$, vacancy, $\mathrm{C}=\mathrm{Ti}, \mathrm{Nb} ; \mathrm{D}=\mathrm{Zr}, \mathrm{Ti} ; \mathrm{T}=\mathrm{Si}$, and $\mathrm{X}=\mathrm{O}, \mathrm{OH}, \mathrm{F}$. There are seven different $A$ sites (Table 5, Figure 5) with coordination numbers varying from $6(A 4)$ and $7(A 3, A 5, A 6)$ to $8(A 1, A 2$, A7) (Table 7) with the average $\langle A$ - $\phi>$ bond lengths $(\phi=\mathrm{O}, \mathrm{OH})$ from 2.369 to $2.520 \AA$. There are one pure $\mathrm{Ca}(A 1)$ and one pure $\mathrm{Na}(A 4)$ sites, whereas the other five $A$ sites have mixed $\mathrm{Na}$-Ca occupancies with different $\mathrm{Na} / \mathrm{Ca}$ ratios (Table 5). The $A 2$ and $A 7$ sites are dominantly occupied by $\mathrm{Ca}$, whereas $\mathrm{Na}$ is the dominant amount in the $A 3, A 5$, and $A 6$ sites. Fe is prevalent in the $B$ site even though it also contains a significant amount of $\mathrm{Mn}$. The $B$ site has an octahedral coordination with the average $\langle B-\phi\rangle$ bond length equal to $2.237 \AA$. The octahedral $C$ site is occupied by Ti with the minor admixture of $\mathrm{Nb}$ with the average $\left\langle C-\phi>\right.$ bond length of $2.011 \AA$. The $\mathrm{CO}_{6}$ octahedra are strongly distorted due to the electronic second-order Jahn-Teller effect [26]. There are one short (1.813 $\mathrm{A})$ and one long (2.209 $\AA$ ) $C-\phi$ apical bonds and four intermediate (1.988-2.031 $\AA$ ) equatorial $C$-O bonds (Table 7). There are two octahedrally coordinated $D$ sites, $D 1$ and $D 2$, which are predominantly occupied by Zr. The $D$ sites have nearly regular octahedral coordinations with the average $\langle D 1-\phi\rangle$ and $\langle D 2-\phi\rangle$ distances of 2.071 and $2.082 \AA$, respectively. The four $T$-sites are occupied by $\mathrm{Si}$ and have tetrahedral coordination with the average $<T$-O $>$ bond lengths ranging from 1.611 to $1.617 \AA$. The $T$-O- $T$ bridges in the $\mathrm{Si}_{2} \mathrm{O}_{7}$ groups are almost linear with $\mathrm{T1}-\mathrm{O} 4-\mathrm{T} 2=166^{\circ}$ and $\mathrm{T} 3-\mathrm{O} 11-\mathrm{T} 4=175^{\circ}$.
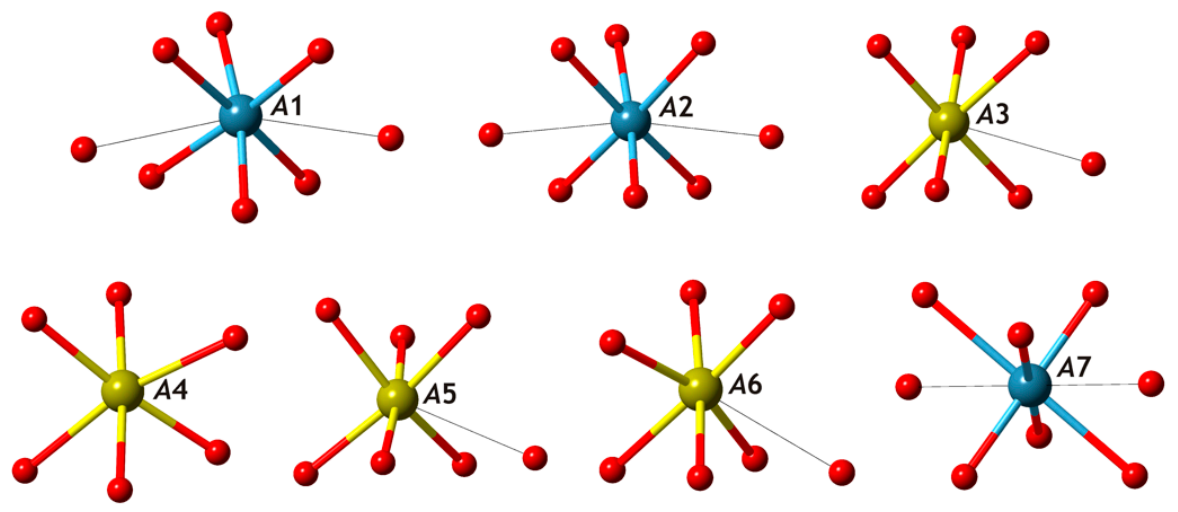

Figure 5. Coordination of the $A$-type cations in the crystal structure of chirvinskyite. The $A$-O bonds longer than $2.6 \AA$ are shown as black single lines. Legend: $\mathrm{Ca}=$ blue, $\mathrm{Na}=$ yellow, and $\mathrm{O}=$ red.

There are six anion X sites, X15-X20, not bonded to Si and occupied by different proportions of $\mathrm{O}$, $\mathrm{OH}$, and F. Taking into account the bond-valence sums and local coordination environments, it can be suggested that the fourfold coordinated $\mathrm{X} 15, \mathrm{X} 17$, and $\mathrm{X} 20$ sites are occupied by $\mathrm{O}^{2-}$ and $\mathrm{F}^{-}$anions, whereas threefold coordinated hydroxyl groups are dominant in the X16, X18, and X19 sites (Table 5). For the ideal end-member formula, the amounts of $\mathrm{O}$ and $\mathrm{OH} / \mathrm{F}$ should be equal, which results in the composition of $\mathrm{Na}_{8} \mathrm{Ca}_{5} \mathrm{Fe}_{2} \mathrm{Ti}_{2} \mathrm{Zr}_{3}\left(\mathrm{Si}_{2} \mathrm{O}_{7}\right)_{4} \mathrm{O}_{6}(\mathrm{OH}, \mathrm{F})_{6}$. 
Chirvinskyite belongs to the new structure type of Ti-Zr disilicates. Its crystal structure can be described in modular terms adopted for the minerals of the wöhlerite family $[3,4]$. From this point of view, the crystal structure of chirvinskyite consists of octahedral walls and $\mathrm{Si}_{2} \mathrm{O}_{7}$ groups (Figure 6). Note that this description is valid in the case that only the $A-\mathrm{O}$ bonds shorter than $2.6 \AA$ are taken into account and, therefore, the coordination of the $A$ sites may be treated as octahedral. There are three kinds of walls consisting of four chains, three chains, and five chains of edge-sharing octahedra (Figure 7). The three-membered and five-membered octahedra walls share a common chain of edge-sharing octahedra to form a continuous layer with a zigzag section parallel to (001). The four-membered walls are linked to the layers by sharing common X15 sites and through the disilicate groups. Each $\mathrm{Si}_{2} \mathrm{O}_{7}$ group is linked to three adjacent octahedral walls. The crystal structure of chirvinskyite considered in this aspect belongs to the new structure type of wallpaper structures that are very common in borates [2,27]. Belov [28] pointed out that wallpaper borates and silicates of the wöhlerite-cuspidine group have much in common in terms of the structure architecture. Therefore, chirvinskyite can be described as a new type of a titanozircono-sorosilicate with the new kind of wallpaper structure. Its structural formula, $\mathrm{Na}_{8} \mathrm{Ca}_{5}(\mathrm{Fe}, \mathrm{Mn})_{2} \mathrm{Ti}_{2}\left(\mathrm{Zr}, \mathrm{Ti}_{3}\left(\mathrm{Si}_{2} \mathrm{O}_{7}\right)_{4} \mathrm{O}_{6}(\mathrm{OH})_{6}\right.$, is in good agreement with the simplified formula determined by the chemical analysis.

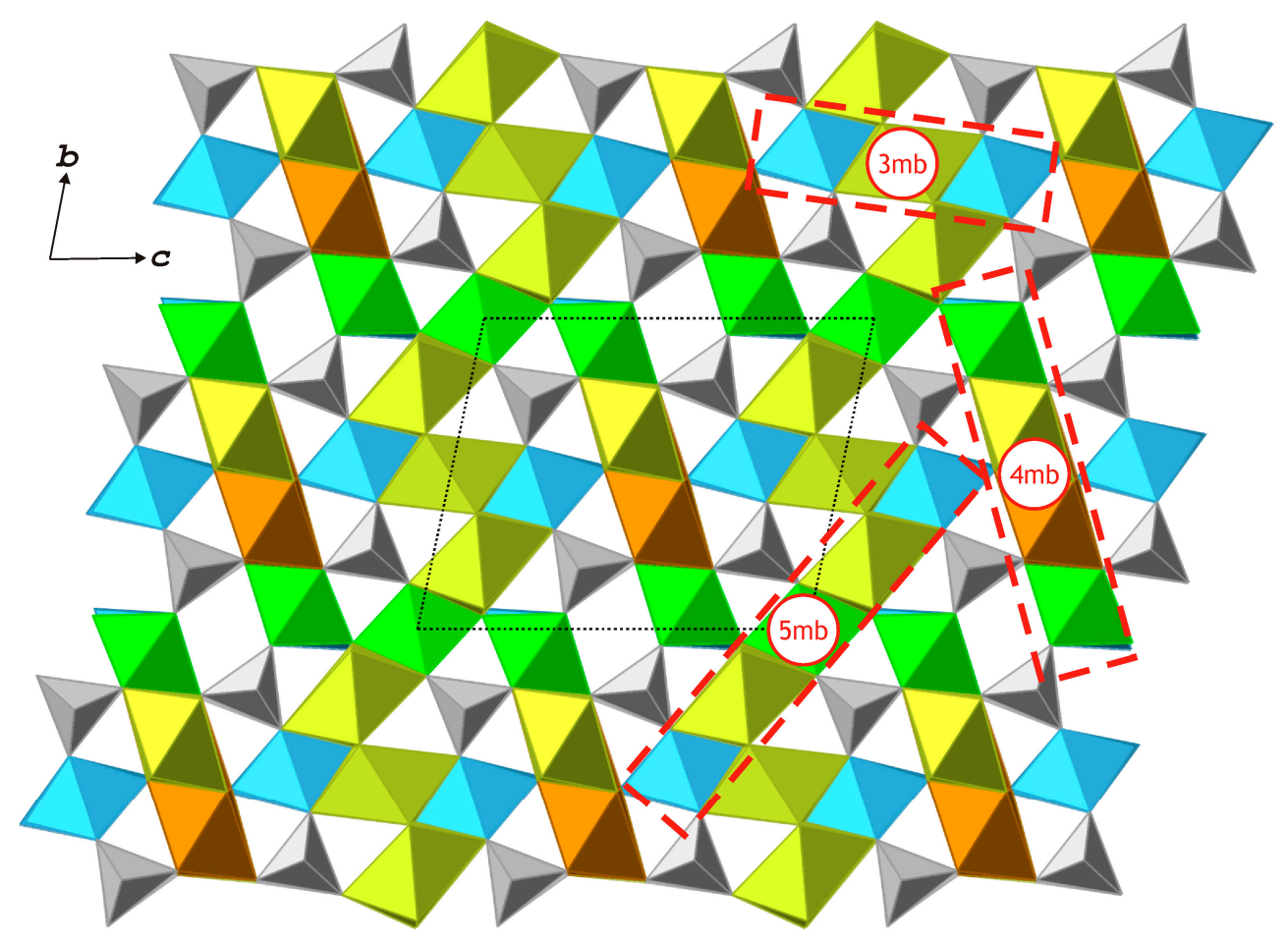

Figure 6. The crystal structure of chirvinskyite projected along the $a$-axis. Red dashed lines outline sections of three-membered, four-membered, and five-membered ( $3 \mathrm{mb}, 4 \mathrm{mb}, 5 \mathrm{mb}$, respectively) octahedral walls. For the $A$ sites, only the $A$-O bonds shorter than $2.6 \AA$ are taken into account. Legend: Ca polyhedra $=$ light-blue, Fe polyhedra $=$ brown, Na polyhedra $=$ yellow, $\mathrm{Zr}$ polyhedra $=$ green, and Si tetrahedra $=$ gray. 
a)

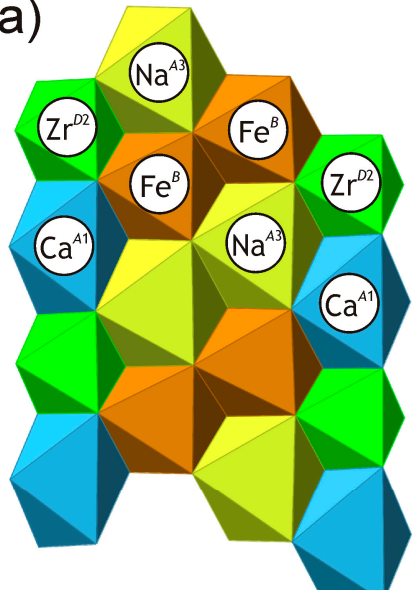

b)

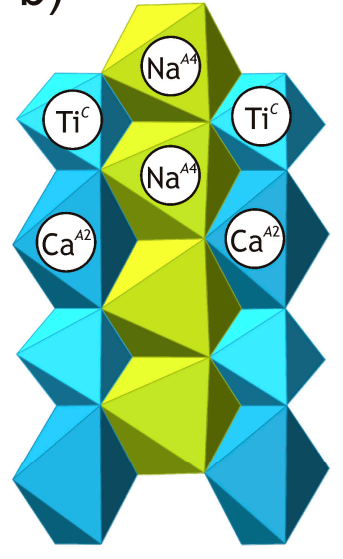

c)

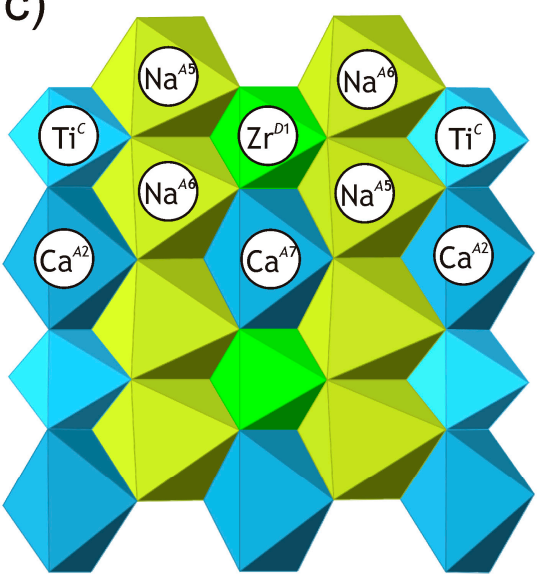

Figure 7. Three types of octahedral walls in the crystal structure of chirvinskyite: four- (a), three- (b) and five- (c) membered. Note that the three-membered and five-membered walls have one octahedral column in common. For the $A$ sites, only the $A-\mathrm{O}$ bonds shorter than $2.6 \AA$ are taken into account. Legend similar to Figure 6.

\section{Discussion}

As already noted, the chirvinskyite-bearing pegmatite initially had the nephelinemicrocline-aegirine-augite composition (with minor arfvedsonite, aenigmatite, astrophyllite, and eudialyte) characteristic for other pegmatites at Mt. Takhtarvumchorr. Later, both pegmatite and host foyaite were metasomatically altered by low-temperature hydrothermal solutions, with replacement of nepheline, microcline, and aegirine-augite by natrolite, albite, and aegirine, respectively.

$$
\begin{gathered}
3 \mathrm{Nph}+\mathrm{Ab}+4 \mathrm{H}_{2} \mathrm{O}=2 \mathrm{Ntr} \\
\mathrm{Mc}+\mathrm{Na}+=\mathrm{Ab}+\mathrm{K}^{+} \\
\text {Aeg-Au }+\mathrm{Na}+=2 \mathrm{Aeg}+\mathrm{Ca}^{2+}
\end{gathered}
$$

where $\mathrm{Ab}$-albite, $\mathrm{NaAlSi}_{3} \mathrm{O}_{8}$, $\mathrm{Aeg}$-aegirine, $\mathrm{NaFeSi}_{2} \mathrm{O}_{6}$, $\mathrm{Aeg}$ - $\mathrm{Au}$-aegirine-augite, $\mathrm{NaCaFe}_{2} \mathrm{Si}_{4} \mathrm{O}_{12}$, $\mathrm{Mc}$-microcline, $\mathrm{KAlSi}_{3} \mathrm{O}_{8}, \mathrm{Nph}$-nepheline, $\mathrm{NaAlSiO}_{4}$, and $\mathrm{Ntr}$-natrolite, $\mathrm{Na}_{2} \mathrm{Al}_{2} \mathrm{Si}_{3} \mathrm{O}_{10} \cdot 2 \mathrm{H}_{2} \mathrm{O}$.

The origin of chirvinskyite can be related to the metasomatic alteration of accessory låvenite by Na-rich hydrothermal solutions, which was accompanied by the replacement of zircon and eudialyte by parakeldyshite and titanite by lorenzenite [5].

$$
\begin{gathered}
\mathrm{Lvt}+4 \mathrm{Ttn}+2 \mathrm{H}_{2} \mathrm{O}+\mathrm{Zr}^{4+}+6 \mathrm{Na}^{+}=\mathrm{Chv}+2 \mathrm{Ti}^{4+}+\mathrm{Ca}^{2+} \\
40 \mathrm{Zrn}+2 \mathrm{Eud}+64 \mathrm{Na}^{+}+7 \mathrm{O}_{2}=46 \mathrm{Pkd}+12 \mathrm{H}_{2} \mathrm{O}+6 \mathrm{Fe}^{2+}+12 \mathrm{Ca}^{2+} \\
2 \mathrm{Ttn}+2 \mathrm{Na}^{+}+\mathrm{H}_{2}=\mathrm{Lor}+\mathrm{H}_{2} \mathrm{O}+2 \mathrm{Ca}^{2+}
\end{gathered}
$$

where Chv—chirvinskyite, $\mathrm{Na}_{8} \mathrm{Ca}_{5} \mathrm{Fe}_{2} \mathrm{Ti}_{2} \mathrm{Zr}_{3} \mathrm{Si}_{8} \mathrm{O}_{34}(\mathrm{OH})_{6}$, Eud-eudialyte, $\mathrm{Na}_{14} \mathrm{Ca}_{6} \mathrm{Fe}_{3} \mathrm{Zr}_{3} \mathrm{Si}_{26} \mathrm{O}_{72}(\mathrm{OH})_{4}$. $4 \mathrm{H}_{2} \mathrm{O}$, Lor-lorenzenite, $\mathrm{Na}_{2} \mathrm{Ti}_{2} \mathrm{Si}_{9} \mathrm{O}_{9}$, Lvt-låvenite, $\mathrm{Na}_{2} \mathrm{Ca}_{2} \mathrm{FeMnZr}_{2} \mathrm{Si}_{4} \mathrm{O}_{16}(\mathrm{OH})_{2}$, $\mathrm{Pkd}$-parakeldyshite, $\mathrm{Na}_{2} \mathrm{ZrSi}_{2} \mathrm{O}_{7}, \mathrm{Ttn}$-titanite, $\mathrm{CaTiSiO}_{5}$, and $\mathrm{Zr}$ - zircon, $\mathrm{ZrSiO}_{4}$. Calcium as a characteristic product of all these reactions is concentrated in fluorapatite.

Under such conditions, there is no wonder that such an $\mathrm{H}_{2} \mathrm{O}$-rich sorosilicate as chirvinskyite was crystallized. This mineral may contain some additional non-structural molecular $\mathrm{H}_{2} \mathrm{O}$, which is detected by the Penfield method and Raman spectroscopy. However, by analogy with similar minerals (such as murmanite, selivanovaite, etc.), the crystal-chemical formula of chirvinskyite does not take these $\mathrm{H}_{2} \mathrm{O}$ molecules into account. 
Chirvinskyite belongs to a new structure type of minerals and inorganic compounds. The related structures are those of låvenite and other "octahedral-wall" titanosilicates. According to the Nickel-Strunz classification, it belongs to the group 9.BE (Sorosilicates with $\mathrm{Si}_{2} \mathrm{O}_{7}$ groups, with additional anions as well as cations in octahedral and greater coordination). According to the Dana classification, it belongs to the group 56 (Sorosilicates with $\mathrm{Si}_{2} \mathrm{O}_{7}$ groups, with additional $\mathrm{O}, \mathrm{OH}$, $\mathrm{F}$, and $\mathrm{H}_{2} \mathrm{O}$ ).

In this study, we define chirvinskyite as a mineral with Fe dominant in the $B$ site. However, large contents of $\mathrm{Mn}$ and $\mathrm{Ca}$ in some aggregates allows us to predict Mn-dominant and vacancy-dominant analogues of chirvinskyite, which await further investigation.

Supplementary Materials: The following are available online at http://www.mdpi.com/2075-163X/9/4/219/s1: Crystallographic Information file (CIF) for the crystal structure of chirvinskyite.

Author Contributions: Field investigation, V.N.Y., G.Y.I., and Y.A.P. Laboratory investigation, V.N.Y., Y.A.P., A.A.Z., T.L.P., J.A.M., and V.N.B. Writing, G.Y.I. and A.A.Z. Supervision and editing, S.V.K.

Funding: The Kola Science Center of Russian Academy of Sciences (Projects 0226-2019-0009, 0226-2019-0051), the Russian Foundation for Basic Research (Grant 18-29-12039), and the Presidium of the Russian Academy of Sciences (Program No 48) funded this research.

Acknowledgments: We are grateful to anonymous reviewers from the CNM NC IMA and MDPI who helped us improve the presentation of our results.

Conflicts of Interest: The authors declare no conflict of interest.

\section{References}

1. Belov, N.V.; Organova, N.I. Crystal chemistry and mineralogy of the lomonosovite group in the light of the crystal structure of lomonosovite. Geokhimiya 1962, 1, 4-13. (In Russian)

2. Grice, J.D.; Burns, P.C.; Hawthorne, F.C. Borate minerals. II. A hierarchy of structures based upon the borate fundamental building block. Can. Mineral. 1999, 37, 731-762.

3. Merlino, S.; Perchiazzi, N. Modular mineralogy in the cuspidine group of minerals. Can. Mineral. 1988, 26, 933-943.

4. Biagioni, C.; Merlino, S.; Parodi, G.C.; Perchiazzi, N. Crystal chemistry of minerals of the wöhlerite group from the Los Archipelago, Guinea. Can. Mineral. 2012, 50, 593-609. [CrossRef]

5. Yakovenchuk, V.N.; Ivanyuk, G.Yu.; Pakhomovsky, Ya.A.; Men'shikov, Yu.P. Khibiny; Wall, F., Ed.; Laplandia Minerals: Apatity, Russia, 2005; ISBN 5900395480.

6. Bohnstedt, E.; Borneman-Starynkevich, I.; Vlodavets, N.; Vorob'eva, O.; Gerasimovsky, V.; Gutkova, N.; Kagan, B.; Kostyleva, E.; Kupletsky, B.; Labuntsov, A.; et al. Minerals of the Khibiny and Lovozero Tundras; AN SSSR Publishing: Moscow, Russia, 1937. (In Russian)

7. Chirvinsky, P.N. On the mineralogy of the Kirovsk region. In Productive Forces of the Kola Peninsula; AN SSSR Publishing: Moscow, Russia, 1940; Volume 1, pp. 27-55. (In Russian)

8. Sandell, E.B. Micro determination of water by the Penfield method. Microchim. Acta 1951, 38, 487-491. [CrossRef]

9. Dolivo-Dobrovolsky, D.D. MINAL, Free Software. Available online: http://www.dimadd.ru (accessed on 8 July 2013).

10. Britvin, S.N.; Dolivo-Dobrovolsky, D.V.; Krzhizhanovskaya, M.G. Software for processing of X-ray powder diffraction data obtained from the curved image plate detector of Rigaku RAXIS Rapid II diffractometer. Zap. Ross. Mineral. Obsh. 2017, 146, 104-107. (In Russian)

11. Bruker AXS GmbH Topas. General Profile and Structure Analysis Software for Powder Diffraction Data; Bruker: Billerica, MA, USA, 2009.

12. APEX2. Version 2014.11-0; Bruker-AXS: Madison, WI, USA, 2014.

13. Sheldrick, G.M. SADABS; University of Goettingen: Goettingen, Germany, 2007.

14. Sheldrick, G.M. Crystal structure refinement with SHELXL. Acta Crystallogr. 2015, C71, 3-8.

15. Sheldrick, G.M. A short history of SHELX. Acta Crystallogr. 2008, A64, 112-122. [CrossRef] [PubMed]

16. Dolomanov, O.V.; Bourhis, L.J;; Gildea, R.J.; Howard, J.A.K.; Puschmann, H. Olex2: A complete structure solution, refinement and analysis program. J. Appl. Crystallogr. 2009, 42, 339-341. [CrossRef] 
17. Atoms 6.5; Shape Software: Kingsport, TN, USA, 2019.

18. Kramm, U.; Kogarko, L.N.; Kononova, V.A.; Vartiainen, H. The Kola alkaline province of the CIS and Finland: Precise Rb-Sr ages define 380-360 Ma age range for all magmatism. Lithos 1993, 30, 33-44. [CrossRef]

19. Korchak, Yu.A.; Men'shikov, Yu.P.; Pakhomovskii, Ya.A.; Yakovenchuk, V.N.; Ivanyuk, G.Yu. Trap formation of the Kola peninsula. Petrology 2011, 19, 87-101. [CrossRef]

20. Yadav, A.K.; Singh, P. A review of structure of oxide glasses by Raman spectroscopy. RSC Adv. 2015, 5, 67583-67609. [CrossRef]

21. Galuskin, E.V.; Lazic, B.; Armbruster, T.; Galuskina, I.O.; Pertsev, N.N.; Gazeev, V.M.; Włodyka, R.; Dulski, M.; Dzierżanowski, P.; Zadov, A.E.; et al. Edgrewite $\mathrm{Ca}_{9}\left(\mathrm{SiO}_{4}\right)_{4} \mathrm{~F}_{2}$-hydroxyledgrewite $\mathrm{Ca}_{9}\left(\mathrm{SiO}_{4}\right)_{4}(\mathrm{OH})_{2}$, a new series of calcium humite-group minerals from altered xenoliths in the ignimbrite of Upper Chegem caldera, Northern Caucasus, Kabardino-Balkaria, Russia. Am. Mineral. 2012, 97, 1998-2006. [CrossRef]

22. Yakovenchuk, V.N.; Krivovichev, S.V.; Ivanyuk, G.Y.; Pakhomovsky, Ya.A.; Selivanova, E.A.; Zhitova, E.S.; Kalashnikova, G.O.; Zolotarev, A.A.; Mikhailova, J.A.; Kadyrova, G.I. Kihlmanite-(Ce), $\mathrm{Ce}_{2} \mathrm{TiO}_{2}\left[\mathrm{SiO}_{4}\right]$ $\left(\mathrm{HCO}_{3}\right)_{2}\left(\mathrm{H}_{2} \mathrm{O}\right)$, a new rare-earth mineral from the pegmatites of the Khibiny alkaline massif, Kola Peninsula, Russia. Mineral. Mag. 2014, 78, 483-496. [CrossRef]

23. Frost, R.L.; López, A.; Xi, Y.; Scholz, R.; Gandini, A.L. A vibrational spectroscopic study of the silicate mineral ardennite-(As). Spectrochim. Acta A Mol. Biomol. Spectrosc. 2014, 24, 987-991. [CrossRef] [PubMed]

24. Libowitzky, E. Correlation of O-H Stretching Frequencies and O-H O Hydrogen Bond Lengths in Minerals. Hydrog. Bond Res. 1999, 1059, 103-115. [CrossRef]

25. Brese, N.E.; O'Keeffe, M. Bond-valence parameters for solids. Acta Crystallogr. 1991, B47, 192-197. [CrossRef]

26. Kunz, M.; Brown, I.D. Out-of-center distortions around octahedrally coordinated d0-transition metals. J. Solid State Chem. 1994, 115, 395-406. [CrossRef]

27. Moore, P.; Araki, T. Pinakiolite, warwikite and wightmanite: Crystal chemistry of complex $3 \AA$ wallpaper structures. Am. Mineral. 1974, 59, 985-1004.

28. Belov, N.V. Essays on Structural Mineralogy; Nedra: Moscow, Russia, 1976. (In Russian)

(C) 2019 by the authors. Licensee MDPI, Basel, Switzerland. This article is an open access article distributed under the terms and conditions of the Creative Commons Attribution (CC BY) license (http://creativecommons.org/licenses/by/4.0/). 